\title{
Basis Function Selection of Frequency-Domain Hammerstein Self-Interference Canceller for In-Band Full-Duplex Wireless Communications
}

\author{
Kazuki Komatsu, Graduate Student Member, IEEE, Yuichi Miyaji, Member, IEEE, \\ and Hideyuki Uehara, Member, IEEE
}

\begin{abstract}
This paper presents a basis function selection technique of a frequency-domain Hammerstein digital selfinterference canceller for in-band full-duplex communications. The power spectral density (PSD) of the nonlinear selfinterference signal is theoretically analyzed in detail, and a nonlinear self-interference PSD estimation method is developed. The proposed selection technique decides on the basis functions necessary for cancellation and relaxes the computational cost of the frequency-domain Hammerstein canceller based on the estimated PSD of the self-interference of each basis function. Furthermore, the convergence performance of the canceller is improved by the proposed selection technique. Simulation results are then presented, showing that the proposed technique can achieve similar cancellation performance compared with the original frequency-domain Hammerstein canceller and a time-domain nonlinear canceller. Additionally, it is shown that the proposed technique improves the computational cost and the convergence performance of the original frequency-domain Hammerstein canceller.
\end{abstract}

Index Terms-Full-duplex radio, self interference, digital cancellation, Hammerstein model.

\section{INTRODUCTION}

I $\mathrm{N}$ recent years there has been a growing interest in inband full-duplex communication systems that involve transmission and reception at the same time and at the same frequency. In theory, full-duplex systems can achieve double the spectral utilization efficiency of conventional half-duplex systems [1]. However, the capacity of full-duplex systems is disturbed by the self-interference caused by simultaneous transmission and reception. To realize a full-duplex system with high spectral efficiency, self-interference cancellation techniques are necessary, and they have been developed in the analog radio frequency (RF) domain, the analog baseband domain, and the digital baseband domain [2].

\section{A. Review of previous research: Analog cancellation}

In general, full-duplex systems can be classified into a shared antenna structure [3] and a two-antenna structure [4]. In a shared antenna structure, the isolation between the transmitter and receiver chain depends on the isolation of the circulator, which can be upwards of $20 \mathrm{~dB}$. In contrast, a two-antenna structure can achieve an isolation of $30 \mathrm{~dB}-50 \mathrm{~dB}$, depending

K. Komatsu, Y. Miyaji, and H. Uehara are with the Department of Electrical and Electronic Information Engineering, Toyohashi University of Technology, Japan (e-mail: komatsu@comm.ee.tut.ac.jp; miyaji@ee.tut.ac.jp; uehara@tut.jp). on the separation distance between the antenna, the direction, and polarization of the antenna [5]. In this paper, we design a self-interference canceller that can be used in either a shared antenna or a two-antenna structure.

Even though attenuation by the antenna is taken into account, self interference still has significant power to saturate the receiver. To avoid receiver saturation, an RF selfinterference canceller is used to reduce the power of the self interference. RF self-interference cancellation techniques have been studied in [1], [3], [4], [6]-[12]. Especially, there are numerous actual experiment reports about the delay-line RF canceller [1], [3], [4], [6], [7], [9], [12]. Thus, in this paper, we assume that the transceiver uses a delay-line canceller as an RF self-interference canceller. The delay-line self-interference canceller is composed of several passive elements such as variable attenuators, phase shifters, and delay lines.

\section{B. Review of previous research: Digital cancellation}

A digital self-interference canceller, which is the final stage of cancellation, is used to adapt to varying wireless channels, and it is necessary to eliminate long delayed and remained self-interference signals. Conventional digital self-interference cancellers can be classified into three categories: time-domain cancellers, frequency-domain cancellers, reference receiver cancellers, and spatial-domain cancellers.

Time-domain digital self-interference cancellers [3], [7], [11]-[16] estimate the self-interference channel, and the self interference is canceled using the estimated channel and the known transmitted signal. In [3], [7], [12]-[16], it is shown that the IQ imbalance of IQ mixers and power-amplifier (PA) nonlinear distortion are serious problems for digital cancellation, and, consequently, nonlinear self-interference cancellers are proposed. A similar idea to nonlinear self-interference cancellers has been studied in the field of acoustic echo cancellation [17], [18]. Time-domain nonlinear cancellers assume the self-interference channel including the transceiver path to be a parallel Hammerstein model, and they have high cancellation characteristics with high computational cost. For example, the least mean squares (LMS) and the recursive least squares (RLS) algorithms for the augmented nonlinear digital canceller [15] need computational costs of $\mathcal{O}\left(P^{2} N\right)$ and $\mathcal{O}\left(P^{4} N^{2}\right)$, respectively, where $P$ is the highest nonlinearity order of the canceller, and $N$ is the number of the taps of each branched finite impulse response (FIR) filter. 
Furthermore, they overestimate nonlinear characteristics even when the power of the self-interference is lower than the noise power because the highest nonlinearity order $P$ is determined so that self-interference can be removed, in the worst case, during the design stage.

Conventional frequency-domain digital self-interference cancellers [4], [19]-[22] estimate the frequency response of the self-interference channel in the frequency domain, and they offer lower computational cost than time-domain cancellers by using fast Fourier transform (FFT). However, they have less flexibility than the time-domain cancellers because they cannot be used with well-known adaptive algorithms such as LMS and RLS, and in [19] the nonlinear coefficients of the PA are estimated by time-domain signal processing. Also, conventional frequency-domain cancellers have several difficulties.

- The performance of the frequency-domain cancellation is degraded when the symbol timing of the desired signal and the self-interference signal are not synchronized [21].

- To avoid the degradation by the symbol timing offset, we have to apply self-interference cancellation on the time domain even though the parameter estimation process is performed on the frequency domain, as in [22].

- When pure OFDM symbols are used for the parameter estimation, the frequency response can be estimated only at the frequency where the subcarriers exist. It may cause degradation of time-domain cancellation performance, which can be found in results of [22] because we have to estimate the effect of discontinuities between symbols which yield the sidelobe of the spectra.

The other category is a canceller based on a reference receiver [23]-[25]. They achieve nonlinear self-interference cancellation with low computational cost and simple algorithms. However, they need an additional auxiliary receiver, and the receiver non-linearities are still to be compensated with it.

On multiple-input and multiple-output (MIMO) full-duplex systems, many papers study spatial-domain cancellation which utilize extra spatial dimensions to cancel self-interference and decode the desired signal. A precoding scheme where the MIMO relay station node optimizes the receive weight matrix and the transmit precoder matrix for reducing self-interference can cancel transmitter-side nonlinearity [26]. In [27], the effect of self-interference is studied theoretically on a massive MIMO relay station node, and it is mentioned that the selfinterference can be reduced by a zero-forcing receiver with a great number of transmitting and receiving antennas.

\section{Our contributions}

Conventional digital self-interference cancellers, which only use a single transmitter-receiver pair, are not suitable to implement on an actual transceiver because they can only achieve high self-interference cancellation at a very high computational cost. In contrast, we have proposed a novel frequency-domain Hammerstein canceller which decreases computational cost by estimating the characteristic of the SI channel on the frequency domain and using the overlap-save method for regenerating SI signals [28]. To the best of our knowledge, the frequency-domain Hammerstein self-interference canceller is the first digital self-interference canceller which estimates non-idealities of the IQ mixers and the PA in the frequencydomain with LMS, RLS, and LS algorithms. However, as with the time-domain Hammerstein canceller, the basis functions used for cancellation are determined in advance, but some of them may be unnecessary when their power is lower than the noise. In addition, the necessary basis functions for some subcarriers may not be needed on another subcarrier. Increasing the number of basis functions leads to an increase in computational cost and convergence time of training, so it is better to reduce the number of basis functions.

- In this paper, we introduce a basis function selection technique for a frequency-domain Hammerstein selfinterference canceller. The proposed selection technique decides basis functions necessary for cancellation and relaxes the computational cost of the frequency-domain Hammerstein canceller based on the power spectral density of the self interference of each basis function.

- In the proposed technique, only the nonlinear characteristics of the transmitter, which can be regarded as static, are learned in advance. To prevent degradation of cancellation performance due to variations in nonlinear characteristics, they are used for the selection of basis functions and not for training self-interference channels.

- Since the proposed technique can be used together with a multi-tap RF canceller, the proposed technique can be applied for much of the full-duplex hardware studied so far.

- Detailed equivalent baseband simulations are performed for verifying the proposed technique, and their results show that the computational cost of the frequency-domain Hammerstein canceller decreases as the power of the self interference decreases. In addition, a canceller with leastsquare training achieves faster convergence characteristics by the proposed selection technique.

Unfortunately, due to specific symbols to train the canceller, the frequency-domain Hammerstein canceller will not be able to track channel variations simultaneously with data transmission. This problem is still not solved in this paper. However, when the symbol timing of the desired signal and the self-interference signal are synchronized, the problem does not arise because specific training symbols are unnecessary. Although this problem is very important, we treat it as a future work in this paper.

The rest of this paper is organized as follows. In Section II, a detailed model of the self interference which includes nonlinearities of the IQ mixers and the power amplifier is provided. The proposed selection technique with the frequencydomain Hammerstein self-interference canceller is presented in Section III. In Section IV, the performance of the proposed technique under different scenarios is analyzed with equivalent baseband signal simulations. Finally, this paper is concluded in Section V. 


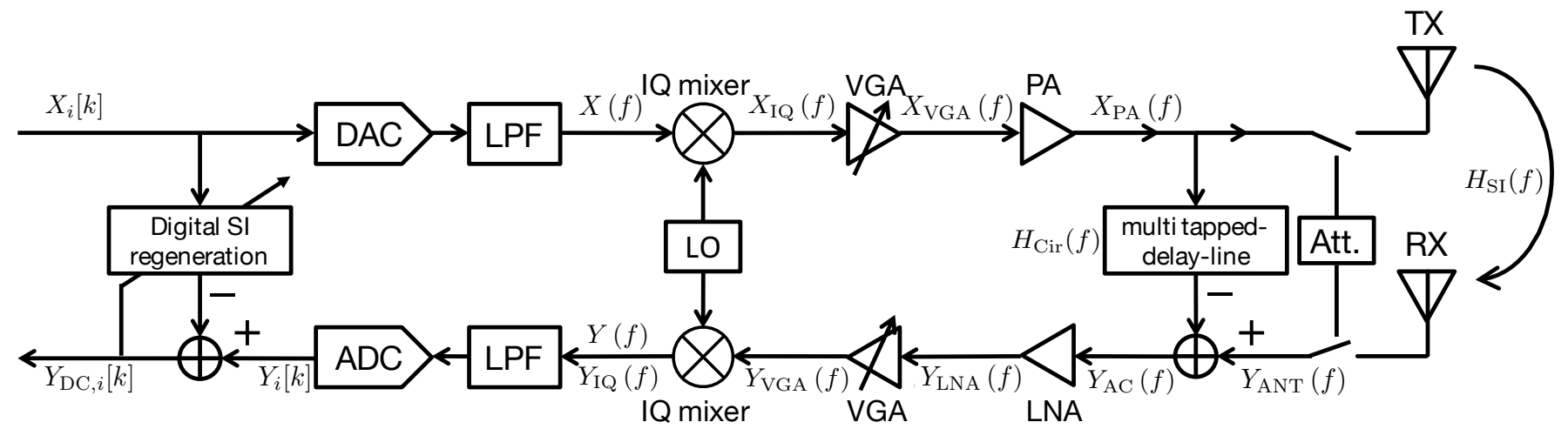

Fig. 1. A model of the assumed full-duplex transceiver.

\section{Self-Interference Signal Model}

Fig. 1 shows the full-duplex direct-conversion transceiver model discussed in this paper. The transceiver consists of a transmitter and a receiver which have IQ mixers, RF filters, and amplifiers. For simplicity, we do not consider the desired signal, and both RF low-pass filters and variable gain amplifiers (VGA) have ideal characteristics. In addition, in the derivation of the mathematical model, we ignore the nonlinear behaviors of the LNA for simplicity, but we assume them on simulations. The nonlinear self-interference signal model has been derived on the time domain in much literature such as [13], [14], [23]. However, we describe the detailed frequencydomain representation of the self interference in this section because we use it to describe our proposed scheme in the next section.

The transceiver transmits an orthogonal frequency division multiplexing (OFDM) signal which has $N_{\mathrm{SC}}$ subcarriers and $N_{\mathrm{CP}}$-length cyclic prefix $(\mathrm{CP})$. The digital-domain discretefrequency transmit signal is expressed as $X_{i}[k]$ where $i$ and $k$ are the symbol index and the subcarrier index, respectively. The transmit signal $X_{i}[k]$ is transformed to time-domain signal $x[n]$ by the OFDM modulator and converted to an analog baseband signal $x(t)$ by an analog to digital converter (ADC) and a low pass filter (LPF). The analog baseband signal $x(t)$ can be expressed as

$$
x(t)=\sum_{\substack{k=-N_{\mathrm{SC}} / 2 \\ k \neq 0}}^{N_{\mathrm{SC}} / 2} X_{i}[k] e^{j 2 \pi k \Delta f t} \quad\left(t \in \mathbf{T}_{i}^{\mathrm{S}} \cup \mathbf{T}_{i}^{\mathrm{CP}}\right),
$$

where $\mathbf{T}_{i}^{\mathrm{S}}$ is the $i$-th symbol duration without $\mathrm{CP}$, and $\mathbf{T}_{i}^{\mathrm{CP}}$ is the $i$-th CP duration, and $\Delta f$ is the frequency interval of each subcarrier. Then we get the frequency-domain representation of the analog baseband transmit signal $X(f)=\mathcal{F}\{x(t)\}$, where $\mathcal{F}\{\cdot\}$ is the operator of the Fourier transform.

\section{A. IQ Mixer}

The analog baseband transmit signal $X(f)$ is upconverted to an RF transmit signal by the IQ mixer of the transmitter. On an ideal IQ mixer, the output equivalent baseband signal of the transmitter IQ mixer $X_{\mathrm{IQ}}(f)$ is equal to the baseband transmit signal $X(f)$. Actually, $X_{\mathrm{IQ}}(f)$ has a mirror-image component of $X(f)$ because an actual IQ mixer has imbalances between the I- and Q-phase carrier signals. The output equivalent baseband signal of the transmitter IQ mixer $X_{\mathrm{IQ}}(f)$ can be expressed as

$$
X_{\mathrm{IQ}}(f)=X(f)+b^{\mathrm{TX}} X^{*}(-f)
$$

where $b^{\mathrm{TX}}$ is the frequency-independent imbalance coefficient of the transmitter IQ mixer, and $(\cdot)^{*}$ denotes the complexconjugate operation. An indicator of the IQ imbalance is called image rejection ratio (IRR) [29], and can be defined as

$$
\mathrm{IRR}^{\mathrm{TX}}=\left|b^{\mathrm{TX}}\right|^{-2} .
$$

Furthermore, the received self-interference signal $Y(f)$ can be expressed as

$$
Y(f)=Y_{\mathrm{IQ}}(f)=Y_{\mathrm{VGA}}(f)+b^{\mathrm{RX}} Y_{\mathrm{VGA}}^{*}(-f),
$$

where $b^{\mathrm{RX}}$ is the frequency-independent imbalance coefficient of the receiver IQ mixer, and $Y_{\mathrm{VGA}}^{*}(f)$ is the equivalent baseband signal of the receiver VGA output signal. As with the transmitter IRR, the receiver IRR is defined as

$$
\mathrm{IRR}^{\mathrm{RX}}=\left|b^{\mathrm{RX}}\right|^{-2} .
$$

\section{B. Power Amplifier}

The output signal of the transmitter IQ mixer $X_{\mathrm{IQ}}(f)$ is amplified by the VGA and the PA of the transmitter because the power of $X_{\mathrm{IQ}}(f)$ is very low for communication with a faraway terminal. Unfortunately, nonlinear distortion of the transmit signal, which is called intermodulation distortion, will occur by nonlinearities of the PA under high transmission power. On time domain, the output signal of the PA is expressed as

$$
\begin{aligned}
x_{\mathrm{PA}}(t) & =h_{\mathrm{PA}}(\tau) *\left(\sum_{p=1,3,5, \ldots}^{\infty} a_{p} x_{\mathrm{IQ}}(t)\left|x_{\mathrm{IQ}}(t)\right|^{p-1}\right) \\
& =h_{\mathrm{PA}}(\tau) *\left(\sum_{p=1,3,5, \cdots}^{\infty} \sum_{q=0}^{p} a_{p} c_{q, p-q} x^{q}(t)\left(x^{*}(t)\right)^{p-q}\right),
\end{aligned}
$$




$$
\begin{aligned}
& c_{p, q}= \begin{cases}0 & ((p<0) \vee(q<0)), \\
\left(b^{\mathrm{TX}}\right)^{q} & ((p, q) \in\{(1,0),(0,1)\}), \\
c_{p, q}^{\prime} & (\text { otherwise }),\end{cases} \\
& c_{p, q}^{\prime}=c_{p-1, q-1}\left(1+\left|b^{\mathrm{TX}}\right|^{2}\right)+c_{p-2, q}\left(b^{\mathrm{TX}}\right)^{*}+c_{p, q-2} b^{\mathrm{TX}}
\end{aligned}
$$

where $h_{\mathrm{PA}}(\tau), a_{p}$, and $x_{\mathrm{IQ}}(t)$ are the impulse response of the $\mathrm{PA}$, the gain of the $p$-th nonlinear distortion, and the timedomain representation of $X_{\mathrm{IQ}}(f)$, respectively. For simplicity, we define the following equations,

$$
\begin{gathered}
\Psi_{p, q}(f)=\mathcal{F}\left\{\psi_{p, q}(t)\right\}=\mathcal{F}\left\{x^{p}(t)\left(x^{*}(t)\right)^{q}\right\}, \\
\sum_{p, q}^{\mathcal{P}}(\cdot)_{p, q}=\sum_{p=1,3,5, \cdots}^{\mathcal{P}} \sum_{q=0}^{p}(\cdot)_{q, p-q},
\end{gathered}
$$

where $\mathcal{P} \in \mathbb{N}$. Thus, the frequency-domain representation of the PA output signal $X_{\mathrm{PA}}(f)$ can be expressed as

$$
X_{\mathrm{PA}}(f)=H_{\mathrm{PA}}(f) \sum_{p, q}^{\infty} a_{p+q} c_{p, q} \Psi_{p, q}(f),
$$

where $H_{\mathrm{PA}}(f)$ is the frequency-domain representation of $h_{\mathrm{PA}}(\tau)$.

\section{Wireless Channel and RF Self-Interference Cancellation}

The signal $X_{\mathrm{PA}}(f)$ is radiated from the transmitter antenna, and is received by the receiver antenna of the same terminal as a strong self interference. The received self-interference signal $Y_{\mathrm{ANT}}(f)$ can be expressed as

$$
Y_{\mathrm{ANT}}(f)=H_{\mathrm{SI}}(f) X_{\mathrm{PA}}(f)+N_{\mathrm{th}}(f),
$$

where $H_{\mathrm{SI}}(f)$ is the frequency-response of the selfinterference channel, $N_{\text {th }}(f)$ denotes thermal noise. The selfinterference channel can be modeled to be a Rician fading channel because the receiver antenna is located close to the transmitter antenna. In [8], it is reported that the Rician factor of the self-interference channel is $20 \mathrm{~dB}<K<40 \mathrm{~dB}$ when the distance between the transmitter antenna and the receiver antenna is 0.5 meters.

The delay-line RF self-interference canceller is composed of several passive elements such as variable attenuators, phase shifters, and delay lines. Therefore, its characteristic can be modeled as a frequency response $H_{\mathrm{Cir}}(f)$. The signal after the RF self-interference cancellation can be expressed as

$$
Y_{\mathrm{AC}}(f)=\left\{H_{\mathrm{SI}}(f)-H_{\mathrm{Cir}}(f)\right\} X_{\mathrm{PA}}(f)+N_{\mathrm{th}}(f),
$$

where the characteristic of $H_{\mathrm{SI}}(f)-H_{\mathrm{Cir}}(f)$ is a strongly frequency-selective channel, because the RF self-interference canceller removes the direct wave and short delay waves of the self-interference signal.

\section{Received Self-Interference Signal}

The input signal of the receiver IQ mixer can be expressed as

$$
\begin{aligned}
Y_{\mathrm{VGA}}(f)= & g_{\mathrm{VGA}}^{\mathrm{RX}} g_{\mathrm{LNA}}^{\mathrm{RX}}\left(H_{\mathrm{SI}}(f)-H_{\mathrm{Cir}}(f)\right) X_{\mathrm{PA}}(f) \\
& +g_{\mathrm{VGA}}^{\mathrm{RX}} g_{\mathrm{LNA}}^{\mathrm{RX}} N_{\text {th }}(f)+N_{\mathrm{LNA}}(f),
\end{aligned}
$$

where $g_{\mathrm{VGA}}^{\mathrm{RX}}, g_{\mathrm{LNA}}^{\mathrm{RX}}$, and $N_{\mathrm{LNA}}(f)$ are the gain of the receiver VGA, the gain of the LNA, and the noise generated by the LNA, respectively. Then the receiver IQ mixer downconverts the signal $Y_{\mathrm{VGA}}(f)$ to an analog baseband signal, and the mirror-image signal of $Y_{\mathrm{VGA}}(f)$ occurs as shown in (4). Therefore, the received analog baseband self-interference signal $Y(f)$ can be expressed as

$$
\begin{aligned}
Y(f) & =H(f) \sum_{p, q}^{\infty} a_{p+q} c_{p, q} \Psi_{p, q}(f) \\
& +b^{\mathrm{RX}} H^{*}(-f) \sum_{p, q}^{\infty} a_{p+q}^{*} c_{p, q}^{*} \Psi_{p, q}^{*}(-f)+N(f), \\
H(f) & =g_{\mathrm{VGA}}^{\mathrm{RX}} g_{\mathrm{LNA}}^{\mathrm{RX}}\left\{H_{\mathrm{SI}}(f)-H_{\mathrm{Cir}}(f)\right\} H_{\mathrm{PA}}(f), \\
N(f) & =\left\{g_{\mathrm{VGA}}^{\mathrm{RX}} g_{\mathrm{LNA}}^{\mathrm{RX}} N_{\mathrm{th}}(f)+N_{\mathrm{LNA}}(f)\right\} \\
& +b^{\mathrm{RX}}\left\{g_{\mathrm{VGA}}^{\mathrm{RX}} g_{\mathrm{LNA}}^{\mathrm{RX}} N_{\mathrm{th}}(-f)+N_{\mathrm{LNA}}(-f)\right\}^{*} .
\end{aligned}
$$

In addition, by the relation $\Psi_{p, q}(f)=\Psi_{q, p}^{*}(-f)$, we can rewrite (14) as

$$
\begin{aligned}
Y(f) & =\sum_{p, q}^{\infty} H_{p, q}(f) \Psi_{p, q}(f)+N(f), \\
H_{p, q}(f) & =a_{p+q} c_{p, q} H(f)+a_{p+q}^{*} b^{\mathrm{RX}} c_{q, p}^{*} H^{*}(-f) .
\end{aligned}
$$

From (17), we get a important fact that the received selfinterference signal $Y(f)$ is a linear combination of $\Psi_{p, q}(f)$ at each frequency.

\section{E. Received Subcarriers}

In the proposed scheme, we use discrete-frequency domain signals which can be generated by $\mathrm{CP}$ removal and discrete Fourier transform (DFT). Thus, it is important to check what kind of signal will occur in the discrete-frequency domain.

The nonlinear distortion signal $\psi_{p, q}(t)$ can be expressed as (19), and we can simplify it as follows:

$$
\psi_{p, q}(t)=\sum_{k=-(p+q) N_{\mathrm{Sc}} / 2}^{(p+q) N_{\mathrm{SC}}} \Psi_{p, q, i}[k] e^{j 2 \pi k \Delta f t}
$$

where $\Psi_{p, q, i}[k]$ denotes a frequency-domain representation of the nonlinear distortion signal $\psi_{p, q}(t)$. Under an assumption that the self-interference channel and the RF canceller do not generate delay signals beyond CPs, the received analog baseband signal $y(t)$ can be expressed as

$$
\begin{aligned}
y(t) & =\sum_{k=-\infty}^{\infty} Y_{i}[k] e^{j 2 \pi k \Delta f t} \quad\left(t \in \mathbf{T}_{i}^{\mathbf{S}}\right), \\
Y_{i}[k] & =\sum_{p, q}^{\infty} H_{p, q}[k] \Psi_{p, q, i}[k]+N_{i}[k], \\
H_{p, q}[k] & =H_{p, q}(k \Delta f)
\end{aligned}
$$

where $N_{i}[k]$ is a narrow-band noise for the $k$-th subcarrier. In (21) and (22), it is notable that we can get $Y_{i}[k]$ by applying the DFT to the received digital baseband signal after removing the $\mathrm{CP}$, and $Y_{i}[k]$ is a linear combination of $\Psi_{p, q, i}[k]$ at each frequency as with (17). Additionally, we can estimate $\mathbb{E}\left[\left|H_{p, q}[k] \Psi_{p, q, i}[k]\right|^{2}\right]$ by (22), and the next section shows how to estimate $\mathbb{E}\left[\left|H_{p, q}[k] \Psi_{p, q, i}[k]\right|^{2}\right]$. 


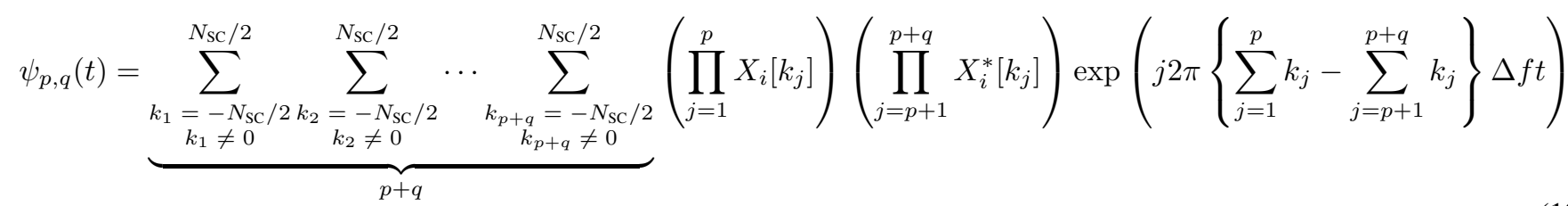

\section{PROPOSED SCHEME}

In this section, we describe the proposed scheme based on a frequency-domain Hammerstein self-interference canceller [28]. The proposed scheme consists of three stages: premeasurement of power ratio, training of the canceller, and cancellation. On the premeasurement stage, we estimate the gain of the $(p, q)$-th nonlinear component to the linear component (GNL) as follows:

$$
G_{p, q}^{\mathrm{N} / \mathrm{L}}=\left|\frac{a_{p+q} c_{p, q}}{a_{1}}\right|,
$$

At the beginning of the training stage, the power of $H_{p, q}[k] \Psi_{p, q, i}[k]$ is estimated based on $G_{p, q}^{\mathrm{N} / \mathrm{L}}[k]$, and we determine whether the $(p, q)$-th basis function is necessary or not. The computational cost can be reduced by the proposed basis function selection scheme because the number of the basis functions used for training is reduced.

\section{A. Premeasurement}

Since the parameters $a_{p+q}$ and $c_{p, q}$ depend on the RF circuits of the transceiver only, they can be assumed to be very static. Thus, we can measure the GNL by a massive computational resource at the time of design or when the terminal is inactive. In addition, we can use a coaxial cable and an attenuator instead of antennas and the RF cancellatin circuit because the GNL does not depend on the frequency response of the self-interference channel and the RF cancellation circuits. Therefore, in this paper, we use a coaxial cable and an attenuator for loopback measurements. When the test OFDM signal $x^{\mathrm{LB}}$ is used to measure the GNL, the received loopback signal can be expressed as

$$
\begin{aligned}
& Y_{i}^{\mathrm{LB}}[k]=\sum_{p, q}^{\infty} H_{p, q}^{\mathrm{LB}}[k] \Psi_{p, q, i}^{\mathrm{LB}}[k]+N_{i}[k], \\
& H_{p, q}^{\mathrm{LB}}[k]=a_{p+q} c_{p, q} H^{\mathrm{LB}}[k]+a_{p+q}^{*} b^{\mathrm{RX}} c_{q, p}^{*}\left(H^{\mathrm{LB}}[-k]\right)^{*}
\end{aligned}
$$

where $\Psi_{p, q, i}^{\mathrm{LB}}[k]$ is the frequency-domain representation of the $(p, q)$-th distorted input signal, and $H_{p, q}^{\mathrm{LB}}[k]$ denotes the loopback frequency response of the transceiver, and $H^{\mathrm{LB}}[k]$ is the frequency response of the coaxial cable and the attenuator, and $N_{i}[k]$ is the noise signal of the $k$-th subcarrier. To measure the GNL, we estimate the loopback frequency-response $H_{p, q}^{\mathrm{LB}}[k]$ of the transceiver. At each subcarrier, we solve the least squares problem as follows:

$$
\begin{array}{r}
\hat{H}_{p, q}^{\mathrm{LB}}[k]=\underset{H_{p, q}^{\mathrm{LB}}[k]}{\arg \min }\left|Y_{i}^{\mathrm{LB}}[k]-\sum_{p, q}^{P} H_{p, q}^{\mathrm{LB}}[k] \Psi_{p, q, i}^{\mathrm{LB}}[k]\right|^{2} \\
\text { for } k \in\left\{-N_{\mathrm{SC}} / 2, \cdots,-1,1, \cdots, N_{\mathrm{SC}} / 2\right\},
\end{array}
$$

where $P$ is the maximum order to estimate distortions. It can be solved by linear least squares algorithm as follows:

$$
\begin{aligned}
\hat{\mathbf{H}}^{\mathrm{LB}}[k] & =\left[\hat{H}_{p, q}^{\mathrm{LB}}[k] \mid(p, q) \leftarrow \mathcal{I}^{P}\right]^{T} \\
& =\left\{\left(\boldsymbol{\Psi}^{\mathrm{LB}}[k]\right)^{H} \boldsymbol{\Psi}^{\mathrm{LB}}[k]\right\}^{-1}\left(\boldsymbol{\Psi}^{\mathrm{LB}}[k]\right)^{H} \mathbf{Y}^{\mathrm{LB}}[k],
\end{aligned}
$$

$$
\begin{aligned}
\mathbf{Y}^{\mathrm{LB}}[k] & =\left[Y_{0}^{\mathrm{LB}}[k], Y_{1}^{\mathrm{LB}}[k], \cdots, Y_{N_{\mathrm{LB}}-1}^{\mathrm{LB}}[k]\right]^{T}, \\
\boldsymbol{\Psi}^{\mathrm{LB}}[k] & =\left[\boldsymbol{\Psi}_{0}^{\mathrm{LB}}[k], \boldsymbol{\Psi}_{1}^{\mathrm{LB}}[k], \cdots, \boldsymbol{\Psi}_{N_{\mathrm{LB}}-1}^{\mathrm{LB}}[k]\right]^{T}, \\
\boldsymbol{\Psi}_{i}^{\mathrm{LB}}[k] & =\left[\Psi_{p, q, i}^{\mathrm{LB}}[k] \mid(p, q) \leftarrow \mathcal{I}^{P}\right]^{T}, \\
\mathcal{I}_{p} & =[(p, 0),(p-1,1), \cdots,(0, p)], \\
\mathcal{I}^{P} & =\left[\mathcal{I}_{1}, \mathcal{I}_{2}, \cdots, \mathcal{I}_{P}\right]
\end{aligned}
$$

where $\left[(\cdot)_{p, q} \mid(p, q) \leftarrow \Lambda\right]=\left[(\cdot)_{\Lambda[0]},(\cdot)_{\Lambda[1]}, \cdots\right]$, and $N_{\mathrm{LB}}$ is the number of OFDM symbols for the premeasurement.

In (18), it is notable that $H_{0,1}[k]$ is a linear combination of $H[k]$ and $H^{*}[-k]$. In addition, we can estimate the channel response $H[k]$ by $H_{1,0}[k] \approx a_{1} c_{1,0} H[k]$ because $\left|c_{1,0}\right| \gg$ $\left|b^{\mathrm{RX}} c_{0,1}\right|$ when $\left|b^{\mathrm{TX}}\right| \ll 1$ and $\left|b^{\mathrm{RX}}\right| \ll 1$. Thus, we can rewrite $H_{0,1}^{\mathrm{LB}}[k]$ as follows:

$$
H_{0,1}^{\mathrm{LB}}[k] \approx b^{\mathrm{TX}} H_{1,0}^{\mathrm{LB}}[k]+b^{\mathrm{RX}}\left(H_{1,0}^{\mathrm{LB}}[-k]\right)^{*} .
$$

$b^{\mathrm{TX}}$ and $b^{\mathrm{RX}}$ can be estimated by following least squares algorithm:

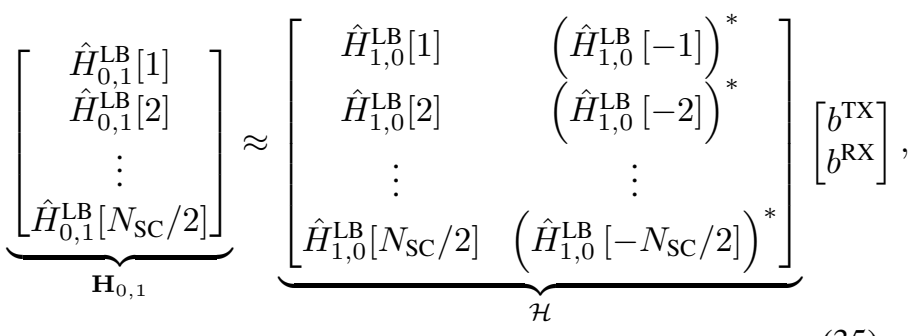

$$
\left[\begin{array}{l}
\hat{b}^{\mathrm{TX}} \\
\hat{b}^{\mathrm{RX}}
\end{array}\right]=\left(\mathcal{H}^{H} \mathcal{H}\right)^{-1} \mathcal{H}^{H} \mathbf{H}_{0,1} .
$$

The receiver IQ imbalance interferes with estimation of the GNL because the GNL is a parameter of the transmitter. Thus, it is necessary to relax the receiver IQ imbalance, and is achieved by the following operation:

$$
\hat{H}_{p, q}^{\mathrm{IQF}}[k]=\frac{\hat{H}_{p, q}^{\mathrm{LB}}[k]-\hat{b}^{\mathrm{RX}}\left(\hat{H}_{q, p}^{\mathrm{LB}}[-k]\right)^{*}}{1-\left|\hat{b}^{\mathrm{RX}}\right|^{2}} .
$$


When $\hat{H}_{p, q}^{\mathrm{LB}}[k]$ and $\hat{b}^{\mathrm{RX}}$ are sufficiently close to the true values, (37) can be approximated as follows:

$$
\begin{aligned}
\hat{H}_{p, q}^{\mathrm{IQF}}[k] & \approx \frac{H_{p, q}^{\mathrm{LB}}[k]-b^{\mathrm{RX}}\left(H_{q, p}^{\mathrm{LB}}[-k]\right)^{*}}{1-\left|b^{\mathrm{RX}}\right|^{2}} \\
& =\frac{a_{p+q} c_{p, q} H^{\mathrm{LB}}[k]-a_{p+q}\left|b^{\mathrm{RX}}\right|^{2} c_{p, q} H^{\mathrm{LB}}[k]}{1-\left|b^{\mathrm{RX}}\right|^{2}} \\
& =a_{p+q} c_{p, q} H^{\mathrm{LB}}[k] .
\end{aligned}
$$

Thus, we can estimate the GNL as follows:

$$
\hat{G}_{p, q}^{\mathrm{N} / \mathrm{L}}=\sqrt{\frac{1}{N_{\mathrm{SC}}} \sum_{\substack{k=-N_{\mathrm{SC}} / 2 \\ k \neq 0}}^{N_{\mathrm{SC}} / 2} \frac{\left|\hat{H}_{p, q}^{\mathrm{IQF}}[k]\right|^{2}}{\left|\hat{H}_{1,0}^{\mathrm{IQF}}[k]\right|^{2}}} .
$$

When $\hat{H}_{p, q}^{\mathrm{LB}}[k]$ and $\hat{b}^{\mathrm{RX}}$ sufficiently are close to the true values, the estimated GNL can be approximated to the true value of the GNL as follows:

$$
\begin{aligned}
\hat{G}_{p, q}^{\mathrm{N} / \mathrm{L}} & \approx \sqrt{\frac{1}{N_{\mathrm{SC}}} \sum_{\substack{k=-N_{\mathrm{SC}} / 2 \\
k \neq 0}}^{N_{\mathrm{SC}} / 2} \frac{\left|a_{p+q} c_{p, q} H^{\mathrm{LB}}[k]\right|^{2}}{\left|a_{1} H^{\mathrm{LB}}[k]\right|^{2}}} \\
& =\sqrt{\frac{1}{N_{\mathrm{SC}}} \sum_{\substack{k=N_{\mathrm{SC}} / 2 \\
k \neq 0}}^{N_{\mathrm{SC} / 2}}\left(G_{p, q}^{\mathrm{N} / \mathrm{L}}\right)^{2}}=G_{p, q}^{\mathrm{N} / \mathrm{L}} .
\end{aligned}
$$

\section{B. Training}

On the training stage, the swapped OFDM modulation, which is introduced in [28], is needed to estimate $H_{p, q}[k]$ out of the band when the symbol timing of the desired signal and the self-interference signal are not synchronized. When the symbol synchronization is achieved, we can use pure OFDM symbols instead of swapped OFDM symbols. In the rest of this paper, we assume unsynchronized situations. The difference between synchronized and unsynchronized situations is whether the swapped OFDM modulation is used instead of pure OFDM modulation and the subcarriers used for the channel estimation. In particular, in synchronized situations, we use pure OFDM symbols and estimate the self-interference channel at $k \in$ $\left\{-N_{\mathrm{SC}} / 2, \cdots,-1,1, \cdots, N_{\mathrm{SC}} / 2\right\}$, and in unsynchronized situations, we use the swapped OFDM symbols and estimate the channel at $k \in\left\{-N_{\mathrm{FFT}} / 2, \cdots,-1,1, \cdots, N_{\mathrm{FFT}} / 2\right\}$ where $N_{\mathrm{FFT}}$ is the FFT size of the OFDM modulation. By the swapped OFDM modulation, subcarriers $X_{i}[k]$ is modulated to a digital baseband signal $x_{i}^{\mathrm{SWP}}[n]$ as follows:

$$
\begin{gathered}
x_{i}[n]=\sum_{\substack{k=-N_{\mathrm{SC}} / 2 \\
k \neq 0}}^{N_{\mathrm{SC}} / 2} X_{i}[k] e^{j 2 \pi k n / N_{\mathrm{FFT}}}\left(n \in\left[0, N_{\mathrm{FFT}}\right)\right), \\
x_{2 i}^{\mathrm{SWP}}=\left[x_{2 i+1}[0], \cdots, x_{2 i+1}\left[N_{\mathrm{FFT}} / 2-1\right]\right. \\
\left.x_{2 i}\left[N_{\mathrm{FFT}} / 2\right], \cdots, x_{2 i}\left[N_{\mathrm{FFT}}-1\right]\right]
\end{gathered}
$$

$$
\begin{aligned}
x_{2 i+1}^{\mathrm{SWP}}=[ & x_{2 i}[0], \cdots, x_{2 i}\left[N_{\mathrm{FFT}} / 2-1\right] \\
& \left.x_{2 i+1}\left[N_{\mathrm{FFT}} / 2\right], \cdots, x_{2 i+1}\left[N_{\mathrm{FFT}}-1\right]\right] .
\end{aligned}
$$

Then the received subcarrier signal $Y_{i}^{\mathrm{SWP}}[k]$ can be expressed as

$$
\begin{aligned}
& Y_{i}^{\mathrm{SWP}}[k]=\sum_{p, q}^{\infty} H_{p, q}[k] \Psi_{p, q, i}^{\mathrm{SWP}}[k]+N_{i}[k], \\
& \Psi_{p, q, i}^{\mathrm{SWP}}[k]=\sum_{n=0}^{N_{\mathrm{FFT}}-1}\left(x_{i}^{\mathrm{SWP}}[n]\right)^{p}\left(x_{i}^{\mathrm{SWP}}[n]\right)^{q} e^{-j 2 \pi \frac{k n}{N_{\mathrm{FFT}}}},
\end{aligned}
$$

where $\Psi_{p, q, i}^{\mathrm{SWP}}[k]$ is the distorted swapped OFDM modulated signal, and $N_{i}[k]$ denotes a noise signal. Before training the canceller parameters, we determine which basis function to use for cancellation at each discrete frequency as follows:

$$
U_{p, q}[k]=\left(\gamma \mathbb{E}\left[\left|H_{p, q}[k] \Psi_{p, q, i}^{\mathrm{SWP}}[k]\right|^{2}\right]>\mathbb{E}\left[\left|N_{i}[k]\right|^{2}\right]\right),
$$

where $U_{p, q}[k]$ denotes whether to use the $(p, q)$-th basis function at the $k$-th subcarrier, $\mathbb{E}\left[\left|(\cdot)_{i}\right|^{2}\right]$ denotes the expected power of $(\cdot)$ for $i$, and $\gamma$ is called the noise margin. In (46), we can control the trade-off between computational cost and accuracy of the self-interference cancellation by the noise margin $\gamma . \mathbb{E}\left[\left|H_{p, q}[k] \Psi_{p, q, i}^{\mathrm{SWP}}[k]\right|^{2}\right]$ cannot estimate directly because we do not know the channel information $H_{p, q}[k]$. So $\mathbb{E}\left[\left|H_{p, q}[k] \Psi_{p, q, i}^{\mathrm{SWP}}[k]\right|^{2}\right]$ is approximated as follows:

$$
\begin{aligned}
\mathbb{E}\left[\left|H_{p, q}[k] \Psi_{p, q, i}^{\mathrm{SWP}}[k]\right|^{2}\right] \leq & P_{p, q}^{(1)}[k]+P_{p, q}^{(2)}[k] \\
& +2 \sqrt{P_{p, q}^{(1)}[k] P_{p, q}^{(2)}[k]},
\end{aligned}
$$

$$
\begin{aligned}
P_{p, q}^{(1)}[k] & =\mathbb{E}\left[\left|a_{p+q} c_{p, q} H[k] \Psi_{p, q, i}^{\mathrm{SWP}}[k]\right|^{2}\right] \\
& =\left(G_{p, q}^{\mathrm{N} / \mathrm{L}}\right)^{2} R_{p, q}[k] \mathbb{E}\left[\left|a_{1} H[k] \Psi_{1,0, i}^{\mathrm{SWP}}[k]\right|^{2}\right], \\
P_{p, q}^{(2)}[k] & =\mathbb{E}\left[\left|a_{p+q}^{*} b^{\mathrm{RX}} c_{q, p}^{*} H^{*}[-k] \Psi_{p, q, i}^{\mathrm{SWP}}[k]\right|^{2}\right] \\
& =\mathbb{E}\left[\left|a_{p+q}^{*} b^{\mathrm{RX}} c_{q, p}^{*} H^{*}[-k]\left(\Psi_{q, p, i}^{\mathrm{SWP}}[-k]\right)^{*}\right|^{2}\right] \\
& =\left|b^{\mathrm{RX}}\right|^{2} P_{q, p}^{(1)}[-k], \\
R_{p, q}[k] & =\frac{\mathbb{E}\left[\left|\Psi_{p, q, i}^{\mathrm{SWP}}[k]\right|^{2}\right]}{\mathbb{E}\left[\left|\Psi_{1,0, i}^{\mathrm{SWP}}[k]\right|^{2}\right]} .
\end{aligned}
$$

In the derivation of (47), we have used the following triangle inequality:

$$
|x+y|^{2} \leq|x|^{2}+|y|^{2}+2|x||y| .
$$

Furthermore, we make (48) easier by the relation of (53) as follows:

$$
P_{p, q}^{(1)}[k] \leq G_{p, q}^{\mathrm{N} / \mathrm{L}} R_{p, q}[k] P_{L}[k] .
$$

Detailed derivation of (53) and (54) is described in Appendix A. Actually, we use (55) instead of $\mathbb{E}\left[\left|Y_{i}^{\mathrm{SWP}}[k]\right|^{2}\right]$ for the basis functions selection because we do not know the true expected spectral density of the received self-interference 


$$
\begin{gathered}
\mathbb{E}\left[\left|a_{1} H[k] \Psi_{1,0, i}^{\mathrm{SWP}}[k]\right|^{2}\right] \leq \mathbb{E}\left[\left|Y_{\mathrm{VGA}, i}^{\mathrm{SWP}}[k]\right|^{2}\right]=\mathbb{E}\left[\left|\frac{Y_{i}^{\mathrm{SWP}}[k]-b^{\mathrm{RX}}\left(Y_{i}^{\mathrm{SWP}}[-k]\right)^{*}}{1-\left|b^{\mathrm{RX}}\right|^{2}}\right|^{2}\right] \leq P_{L}[k] \\
P_{L}[k]=\left.\left.|1-| b^{\mathrm{RX}}\right|^{2}\right|^{-2}\left(\mathbb{E}\left[\left|Y_{i}^{\mathrm{SWP}}[k]\right|^{2}\right]+\left|b^{\mathrm{RX}}\right|^{2} \mathbb{E}\left[\left|Y_{i}^{\mathrm{SWP}}[-k]\right|^{2}\right]+2\left|b^{\mathrm{RX}}\right| \sqrt{\mathbb{E}\left[\left|Y_{i}^{\mathrm{SWP}}[k]\right|^{2}\right] \mathbb{E}\left[\left|Y_{i}^{\mathrm{SWP}}[-k]\right|^{2}\right]}\right)
\end{gathered}
$$

signal. Using $N_{\mathrm{EP}}$ symbols for estimation received signal power spectral density, we estimate $\mathbb{E}\left[\left|Y_{i}^{\mathrm{SWP}}[k]\right|^{2}\right]$ by

$$
\mathbb{E}\left[\left|Y_{i}^{\mathrm{SWP}}[k]\right|^{2}\right] \approx \tilde{P}_{Y}[k]=\frac{1}{N_{\mathrm{EP}}} \sum_{i=0}^{N_{\mathrm{EP}}-1}\left|Y_{i}^{\mathrm{SWP}}[k]\right|^{2} .
$$

In the proposed scheme, we do not estimate and use $\left|b^{\mathrm{RX}}\right|$ to estimate (49) while we can easily estimate $\left|b^{\mathrm{RX}}\right|$ just like $G_{p, q}^{\mathrm{N} / \mathrm{L}}$. The reason for this decision is that (47) and (54) are very sensitive to $\left|b^{\mathrm{RX}}\right|$ on frequency-selective self-interference channels after analog cancellation. In other word, the behavior of (47) and (54) greatly changes depending on whether or not $P_{p, q}^{(1)}[k] \approx\left|b^{\mathrm{RX}}\right|^{2} P_{q, p}^{(1)}[-k]$ and $\mathbb{E}\left[\left|Y_{i}^{\mathrm{SWP}}[k]\right|^{2}\right] \approx$ $\left|b^{\mathrm{RX}}\right|^{2} \mathbb{E}\left[\left|Y_{i}^{\mathrm{SWP}}[-k]\right|^{2}\right]$ are satisfied respectively, and it may lead to deterioration of cancellation performance. To solve this problem, we introduce $\beta$ as a tuning parameter instead of $\left|b^{\mathrm{RX}}\right|$. By setting $\beta$ to be greater than $\left|b^{\mathrm{RX}}\right|$, we can avoid deterioration while computational cost increases.

To summarize the above, we can determine which basis functions to use for self-interference channel estimation by (56)-(58),

$$
\begin{aligned}
\mathcal{J}[k] & =\left[(p, q) \mid \gamma \tilde{P}_{p, q}[k]>\tilde{P}_{N},(p, q) \leftarrow \mathcal{I}^{P}\right] \\
\tilde{P}_{p, q}[k] & =\left(G_{p, q}^{\mathrm{N} / \mathrm{L}}\right)^{2} R_{p, q}[k] \tilde{P}_{L}[k]+\beta^{2}\left(G_{q, p}^{\mathrm{N} / \mathrm{L}}\right)^{2} R_{q, p}[-k] \tilde{P}_{L}[-k] \\
& +\beta G_{p, q}^{\mathrm{N} / \mathrm{L}} G_{q, p}^{\mathrm{N} / \mathrm{L}} \sqrt{R_{p, q}[k] R_{q, p}[-k] \tilde{P}_{L}[k] \tilde{P}_{L}[-k]},
\end{aligned}
$$$$
\tilde{P}_{L}[k]=\frac{\tilde{P}_{Y}[k]+\beta^{2} \tilde{P}_{Y}[-k]+2 \beta \sqrt{\tilde{P}_{Y}[k] \tilde{P}_{Y}[-k]}}{\left|1-\beta^{2}\right|^{2}} .
$$

where $\mathcal{J}[k]$ indicates the set of selected basis functions for each subcarrier, and $\tilde{P}_{N}$ is the estimated expected power of the additive white Gaussian noise $N_{i}[k]$.

Then, we estimate the self-interference channel $H_{p, q}[k]$ independently at each subcarrier to reconstruct the self interference. Since it can be expected that the power of the basis function not included in $\mathcal{J}[k]$ is smaller than the noise, the received training signal can be represented by a linear combination of the basis function constituting $\mathcal{J}[k]$ as

$$
Y_{i}^{\mathrm{SWP}}[k]=\sum_{(p, q) \in \mathcal{J}[k]} H_{p, q}[k] \Psi_{p, q, i}^{\mathrm{SWP}}[k]+N^{\prime}[k],
$$

where $N^{\prime}[k]$ is the sum of the received noise and the nonlinear components determined to be unnecessary for self-interference channel estimation.
Thus, the transmit symbol vector and the channel vector can be defined as (60) and (61), respectively.

$$
\begin{aligned}
\boldsymbol{\Psi}_{i}^{\mathrm{SWP}}[k] & =\left[\Psi_{p, q, i}^{\mathrm{SWP}}[k] \mid(p, q) \leftarrow \mathcal{J}[k]\right]^{T}, \\
\mathbf{H}[k] & =\left[H_{p, q}[k] \mid(p, q) \leftarrow \mathcal{J}[k]\right]^{T} .
\end{aligned}
$$

The channel response $\mathbf{H}[k]$ can be estimated by well-known estimation algorithms such as least squares (LS) algorithms, recursive least squares (RLS) algorithms, and normalized least mean squares (NLMS) algorithms.

1) Least squares algorithm: To apply the LS algorithm to estimate $\mathbf{H}[k]$, we introduce the received symbol vector and the transmit symbol matrix as (62) and (63), respectively.

$$
\begin{aligned}
& \mathbf{Y}^{\mathrm{SWP}}[k]=\left[\begin{array}{lllll}
Y_{0}^{\mathrm{SWP}}[k] & Y_{1}^{\mathrm{SWP}}[k] & \cdots & Y_{N_{\mathrm{Tr}}-1}^{\mathrm{SWP}}[k]
\end{array}\right]^{T} \\
& =\mathbf{\Psi}^{\mathrm{SWP}}[k] \mathbf{H}[k]+\mathbf{N}[k] \\
& \boldsymbol{\Psi}^{\mathrm{SWP}}[k]=\left[\begin{array}{lllll}
\boldsymbol{\Psi}_{0}^{\mathrm{SWP}}[k] & \boldsymbol{\Psi}_{1}^{\mathrm{SWP}}[k] & \cdots & \boldsymbol{\Psi}_{N_{\mathrm{Tr}}-1}^{\mathrm{SWP}}[k]
\end{array}\right]^{T} \\
& \mathbf{N}[k]=\left[\begin{array}{llll}
N_{0}^{\prime}[k] & N_{1}^{\prime}[k] & \cdots & N_{N_{\mathrm{Tr}}-1}^{\prime}[k]
\end{array}\right]^{T}
\end{aligned}
$$

Now, we get the least squares estimated channel $\widehat{H}_{p, q}[k]$ of (62) as

$$
\begin{aligned}
\widehat{\mathbf{H}}[k] & =\left[\widehat{H}_{p, q}[k] \mid(p, q) \leftarrow \mathcal{J}[k]\right]^{T} \\
& =\left\{\left(\boldsymbol{\Psi}^{\mathrm{SWP}}[k]\right)^{H} \mathbf{\Psi}^{\mathrm{SWP}}[k]\right\}^{-1}\left(\boldsymbol{\Psi}^{\mathrm{SWP}}[k]\right)^{H} \mathbf{Y}^{\mathrm{SWP}}[k] .
\end{aligned}
$$

2) Recursive least squares algorithm: Since the least squares method requires matrix inversion or singular value decomposition (SVD), it may be too complicated to actually implement. On the other hand, the RLS algorithm can recursively estimate the self-interference channel which minimizes least square errors without matrix inversion and SVD. On the proposed scheme, the RLS algorithm is expressed as

$$
\begin{aligned}
\widehat{\mathbf{H}}_{i}[k] & =\widehat{\mathbf{H}}_{i-1}[k]+E_{i}[k] \mathbf{G}_{i}[k], \\
E_{i}[k] & =Y_{i}[k]-\left(\mathbf{\Psi}_{i}^{\mathrm{SWP}}[k]\right)^{T} \widehat{\mathbf{H}}_{i-1}[k], \\
\mathbf{G}_{i}[k] & =\frac{\mathbf{P}_{i-1}[k]\left(\mathbf{\Psi}_{i}^{\mathrm{SWP}}[k]\right)^{*}}{\lambda+\left(\mathbf{\Psi}_{i}^{\mathrm{SWP}}[k]\right)^{T} \mathbf{P}_{i-1}[k]\left(\mathbf{\Psi}_{i}^{\mathrm{SWP}}[k]\right)^{*}}, \\
\mathbf{P}_{i}[k] & =\lambda^{-1}\left\{\mathbf{P}_{i-1}[k]-\mathbf{G}_{i}[k]\left(\mathbf{\Psi}_{i}^{\mathrm{SWP}}[k]\right)^{T} \mathbf{P}_{i-1}[k]\right\},
\end{aligned}
$$

where $\widehat{\mathbf{H}}_{i}[k]$ is the $k$-th estimated frequency response on the $i$ th iteration, and $\lambda$ is called forgetting factor. At the beginning of RLS algorithm, $\widehat{\mathbf{H}}_{i}[k]$ and $\mathbf{P}_{i}[k]$ are initialized to $\mathbf{0}$ and $\delta^{-1} \mathbf{I}$ where $\delta$ is very small positive value. The total number 
of multiplications of complex value for computing (66)-(69) on each discrete frequency is $4|\mathcal{J}[k]|^{2}+4|\mathcal{J}[k]|$, where $|\mathcal{J}[k]|$ is the number of elements constituting $\mathcal{J}[k]$.

3) Normalized least mean squares algorithm: The NLMS algorithm, which has a much lower computational cost, is often used when the computational cost of the RLS algorithm produces problems such as processing speed. In the proposed scheme, the NLMS algorithm is expressed as

$$
\begin{aligned}
\widehat{\mathbf{H}}_{i}[k] & =\widehat{\mathbf{H}}_{i-1}[k]+\frac{\mu}{Q_{i}[k]} E_{i}[k]\left(\boldsymbol{\Psi}_{i}^{\mathrm{SWP}}[k]\right)^{*}, \\
Q_{i}[k] & =\left(\boldsymbol{\Psi}_{i}^{\mathrm{SWP}}[k]\right)^{H}\left(\boldsymbol{\Psi}_{i}^{\mathrm{SWP}}[k]\right), \\
E_{i}[k] & =Y_{i}[k]-\left(\boldsymbol{\Psi}_{i}^{\mathrm{SWP}}[k]\right)^{T} \widehat{\mathbf{H}}_{i-1}[k],
\end{aligned}
$$

where $\mu$ is positive constant values. The total number of multiplications of complex values for computing (70)-(72) is $2|\mathcal{J}[k]|$, and it is at least $N$ times faster than the time-domain NLMS algorithm, where $N$ is the number of taps of a timedomain FIR filter.

4) Computational cost of the training stage: At the beginning of the training stage, the set of the basis functions $\mathcal{J}[k]$ is determined on each discrete frequency by (55)(58). In (55)-(58), the most complicated computation is the square root, which must be computed twice at each discrete frequency. Since it is only necessary once at the beginning of the training stage, the computational cost of (55)-(58) is sufficiently smaller than channel estimation which processes for each symbol.

In the channel estimation process of the proposed scheme, received OFDM symbols are decomposed to discretefrequency components by FFT to get (59), and it requires $\frac{1}{2} N_{\mathrm{FFT}} \log _{2} N_{\mathrm{FFT}}$ times multiplications of two complex numbers per symbol. In this paper, we assume $(x[n])^{p}\left(x^{*}[n]\right)^{q}$ can be computed a priori and implemented by a lookup table. Then, the transmitted symbols are distorted by $(x[n])^{p}\left(x^{*}[n]\right)^{q}$ and also decomposed to discrete-frequency components by FFT. The total computational cost of transforming symbols to the frequency domain is $\frac{1}{4}\left(\left|\mathcal{I}^{P}\right|+\right.$ 2) $N_{\mathrm{FFT}} \log _{2} N_{\mathrm{FFT}}$ per symbol because FFT $\left\{(x[n])^{q}\left(x^{*}[n]\right)^{p}\right\}$ can be computed by frequency-inversion and conjugation of FFT $\left\{(x[n])^{p}\left(x^{*}[n]\right)^{q}\right\}$. Next, the self-interference channel $H_{p, q}[k]$ is estimated by estimation algorithms based on $\mathcal{J}[k]$. The NLMS algorithm and the RLS algorithm require $2|\mathcal{J}[k]|$ and $4|\mathcal{J}[k]|^{2}+4|\mathcal{J}[k]|$ complex multiplications per discrete frequency per symbol, respectively. Thus, the whole computational cost of the channel estimation process is

$$
\frac{\frac{1}{4}\left(\left|\mathcal{I}^{P}\right|+2\right) N_{\mathrm{FFT}} \log _{2} N_{\mathrm{FFT}}+\sum_{k=-N_{\mathrm{FFT}} / 2}^{N_{\mathrm{FFT}} / 2} A[k]}{N_{\mathrm{FFT}}+N_{\mathrm{CP}}} \text { per sample, }
$$

where $A[k]$ is $2|\mathcal{J}[k]|$ (for NLMS case) or $4|\mathcal{J}[k]|^{2}+4|\mathcal{J}[k]|$ (for RLS case).

\section{Self-interference reconstruction}

After the training period, we reconstruct the received self interference from the transmit signal and channel information.
We apply cancellation on the time domain because performance of the frequency-domain cancellation is degraded by the symbol timing offset. To reduce the computational cost of the time-domain reconstruction, the overlap-save algorithm [30] is used to reconstruct the received self interference, like [28]. By the overlap-save algorithm, the received self interference can be reconstructed as

$$
y_{\mathrm{RG}}\left[m N_{\mathrm{OLS}}+l\right]=\operatorname{IFFT}\left\{Y_{m}^{\prime}\right\}\left[N_{\mathrm{CP}}+l\right],
$$

where

$$
\begin{gathered}
Y_{m}^{\prime}[k]=\sum_{(p, q) \in \mathcal{J}[k]} \widehat{H}_{p, q}[k] \Psi_{p, q, m}^{\prime}[k], \\
\Psi_{p, q, m}^{\prime}[k]=\operatorname{FFT}\left\{\psi_{p, q}\left[m N_{\mathrm{OLS}}-N_{\mathrm{CP}}\right], \cdots,\right. \\
\left.\psi_{p, q}\left[(m+1) N_{\mathrm{OLS}}-1\right]\right\}[k], \\
\psi_{p, q}[n]=(x[n])^{p}\left(x^{*}[n]\right)^{q},
\end{gathered}
$$

and $N_{\mathrm{OLS}}=N_{\mathrm{FFT}}-N_{\mathrm{CP}}, l \in\left[0, N_{\mathrm{OLS}}\right)$, and $x[n]$ is the transmit baseband signal. In addition, FFT $\{\cdot\}$ and IFFT $\{\cdot\}$ are fast fourier transform operations with an $N_{\mathrm{FFT}}$-size signal. Then, we can get the digital self-interference canceled signal $y_{\mathrm{DC}}[n]$ by subtracting the reconstructed signal $y_{\mathrm{RG}}[n]$ from received signal $y[n]$.

$$
y_{\mathrm{DC}}[n]=y[n]-y_{\mathrm{RG}}[n]
$$

\section{Numerical Simulations}

In this section, we provide numerical simulation results to verify the proposed scheme. To show the effectiveness of the proposed scheme, we compare the performance of the proposed scheme with a conventional scheme.

\section{A. Simulation environment}

To verify the proposed scheme, equivalent baseband simulations of the full-duplex transceiver shown in Fig. 1 is performed. The baseband signal simulator is implemented with the $\mathrm{D}$ programming language, where each non-ideality is modeled with realistic behaviors. In this simulation, we set parameters to the values in TABLE I, which are based on [14]. Since the dynamic range of the receiver ADC is about $79 \mathrm{~dB}$ under these simulation parameters [33], the largest barrier to self-interference cancellation is nonlinear distortions of IQ mixers and the PA. The PA nonlinearities are realized by the Rapp model [34], [35], which is often used to simulate class AB solid state power amplifiers. The output baseband signal of the Rapp modeled PA is described as

$$
\begin{aligned}
y & =G_{\mathrm{PA}} \Gamma(|x|) \frac{x}{|x|}, \\
\Gamma(|x|) & =|x|\left(1+\left(\frac{|x|}{V_{\mathrm{sat}}}\right)^{2 S_{\mathrm{PA}}}\right)^{-\frac{1}{2 S_{\mathrm{PA}}}},
\end{aligned}
$$

where $x$ and $y$ are the input and output signals of the $\mathrm{PA}$, respectively. The Rapp model is characterized by the smoothness factor $S_{\mathrm{PA}}$, the saturation voltage $V_{\text {sat }}$, and the gain $G_{\mathrm{PA}}$. In this paper, we use $S_{\mathrm{PA}}=1$ because we assume there are no linearization techniques of the PA on the transceiver, 
TABLE I

SIMULATION SPECIFICATIONS

\begin{tabular}{|c|c|}
\hline Parameter & Value \\
\hline Oversampling rate & 4 \\
\hline Modulation & OFDM \\
\hline Constellation & 16QAM \\
\hline Size of FFT $N_{\mathrm{FFT}}$ & 256 \\
\hline \# of subcarriers & 52 \\
\hline Cyclic prefix $N_{\mathrm{CP}}$ & 64 samples \\
\hline Sampling rate & $80 \mathrm{M}$ samples/sec \\
\hline Transmit data & Uniform-random data \\
\hline SI channel after RF-SIC & Rayleigh fading \\
\hline SI channel length & 64 taps \\
\hline Transmit power & $23 \mathrm{dBm}$ \\
\hline IRR & $25 \mathrm{~dB}$ \\
\hline PA IIP3 & $21.8 \mathrm{dBm}$ based on [31] \\
\hline PA Gain & $28.5 \mathrm{~dB}$ based on [31] \\
\hline PA smoothness factor, $S_{\mathrm{PA}}$ & 1 \\
\hline LNA IIP3 & $-3 \mathrm{dBm}$ based on [32] \\
\hline LNA smoothness factor, $S_{\mathrm{LNA}}$ & 1,3 \\
\hline Noise figure of receiver & $4 \mathrm{~dB}$ \\
\hline$\#$ of ADC bits & 14 bits \\
\hline Highest order of basis function $P$ & 3 \\
\hline$\#$ of symbols for (55), $N_{\mathrm{EP}}$ & 2 \\
\hline$\lambda$ of the RLS & 1 \\
\hline$\delta$ of the RLS & $\begin{array}{ll}3 \times 10^{-3} & \text { for time-domain } \\
3 \times 10^{-7} & \text { for freq.-domain }\end{array}$ \\
\hline$\mu$ of the NLMS & $\begin{array}{ll}0.2 & \text { for time-domain } \\
0.8 & \text { for freq.-domain }\end{array}$ \\
\hline \# of taps for time-domain scheme & 64 \\
\hline Trials & 101 \\
\hline
\end{tabular}

and the other parameters are set according to the IIP3 and the gain in TABLE I. In addition, we simulate the nonlinearities of the LNA by the Rapp model with smoothness factor $S_{\mathrm{LNA}} \in\{1,3\}$ and IIP $=-3 \mathrm{dBm}$. The case of $S=1$ assume stronger nonlinearities than the case of $S_{\mathrm{LNA}}=3$.

The simulation model of IQ mixers has IQ imbalance achieved by adding an image signal, and its coefficients are predetermined based on the value of IRR. The parameters of both the RLS algorithm and the NLMS algorithm are set to the optimal coefficient which can achieve the best steady-state cancellation performance in each canceller. This condition ensures that the simulation results show the true performance of each canceller. Following the standard convention in literature, the self-interference cancellation ratio (SICR) is defined as

$$
\mathrm{SICR}=\frac{\mathbb{E}\left[|y[n]|^{2}\right]}{\mathbb{E}\left[\left|y_{\mathrm{DC}}[n]\right|^{2}\right]} .
$$

Additionally, we define the self interference to noise power ratio (INR) as

$$
\mathrm{INR}=\frac{\mathbb{E}\left[|y[n]-z[n]|^{2}\right]}{\mathbb{E}\left[|z[n]|^{2}\right]},
$$

where $z[n]$ is the received total noise on the digital baseband. Each simulation result shows the median value of 100 trials. In addition, we calculate the complex multiplication operations per sample (CMOPS), which indicates the number of complex multiplications to process one sample, as the computational cost. For example, the CMOPS of the timedomain Hammerstein canceller optimized by the RLS algo-

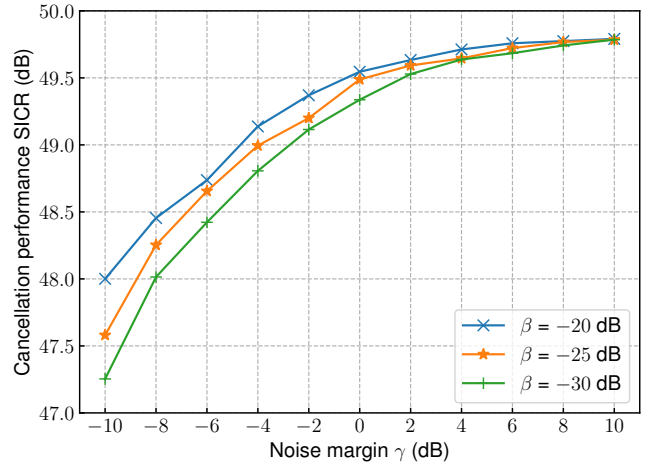

Fig. 2. The median SICR of the proposed scheme at $-10 \mathrm{~dB} \leq \gamma \leq 10 \mathrm{~dB}$ with INR $=50 \mathrm{~dB}$. The RLS algorithm is used as the training algorithm. The smoothness factor of the LNA is $S_{\mathrm{LNA}}=3$.

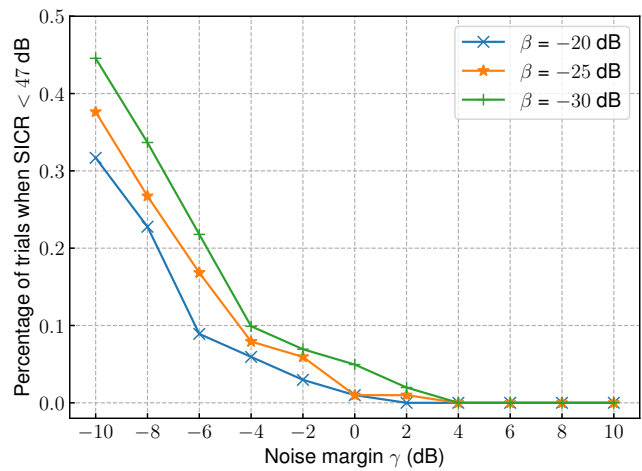

Fig. 3. The percentage of trails where the SICR of the proposed scheme is lower than $47 \mathrm{~dB}$ in all trials at $-10 \mathrm{~dB} \leq \gamma \leq 10 \mathrm{~dB}$ with $\mathrm{INR}=50 \mathrm{~dB}$. The RLS algorithm is used as the training algorithm. The smoothness factor of the LNA is $S_{\mathrm{LNA}}=3$.

rithm with 64-taps FIR filters and six basis functions such as $\left\{x, x^{*},(x)^{3}, x|x|^{2}, x^{*}|x|^{2},\left(x^{*}\right)^{3}\right\}$ is

$$
\begin{aligned}
\mathrm{CMOPS}_{\mathrm{PH}-\mathrm{RLS}} & =4 \times(6 \times 64)^{2}+4 \times(6 \times 64) \\
& \approx 5.91 \times 10^{5}
\end{aligned}
$$

because they update $6 \times 64$ coefficients by the RLS algorithm on each sample. In the same way, the CMOPS of the timedomain Hammerstein canceller optimized by the NLMS algorithm is

$$
\mathrm{CMOPS}_{\mathrm{PH}-\mathrm{NLMS}}=2 \times(6 \times 64)=768 .
$$

\section{B. Results and discussion}

Fig. 2 shows the median cancellation performance of all trials with different $\beta$ and $\gamma$ under IRR of $25 \mathrm{~dB}$ and INR of $50 \mathrm{~dB}$. In addition, Fig. 3 shows the ratio of all trials to the trial where the proposed scheme achieves a SICR of less than $47 \mathrm{~dB}$ on the same situation as Fig. 2. In this scenario, we use the RLS algorithm as a estimation algorithm, and Fig. 4 shows the CMOPS of the proposed scheme at the training stage. In addition, the number of the training symbols $N_{\text {SWP }}$ is 60 to train the canceller completely. Looking at (56)-(58), it is easy to see that increasing the noise margin $\gamma$ increases the number of elements of $\mathcal{J}[k]$. It involves higher cancellation performance and higher computational cost, which can be 


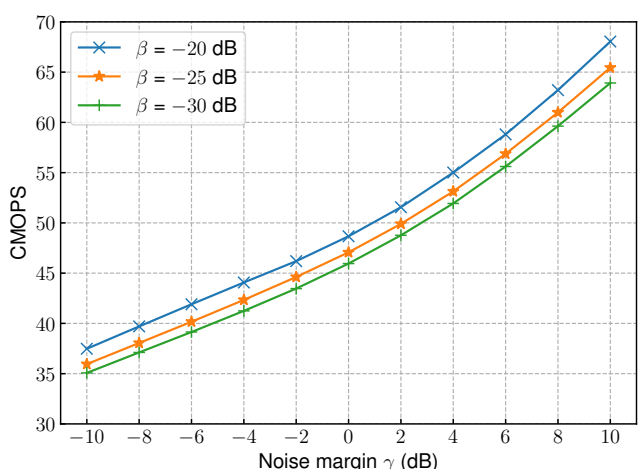

Fig. 4. The computational cost of the proposed scheme at $-10 \mathrm{~dB} \leq \gamma \leq$ $10 \mathrm{~dB}$ with INR $=50 \mathrm{~dB}$. The RLS algorithm is used as the training algorithm. The smoothness factor of the LNA is $S_{\mathrm{LNA}}=3$.

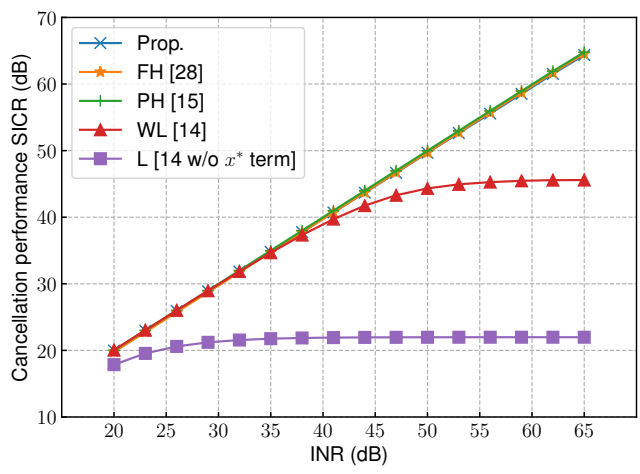

Fig. 5. The median SICR value of each canceller with the LS algorithm at $N_{\operatorname{Tr}}=60$. The smoothness factor of the LNA is $S_{\mathrm{LNA}}=3$. For the proposed scheme, $\beta=-20 \mathrm{~dB}$ and $\gamma=0 \mathrm{~dB}$.

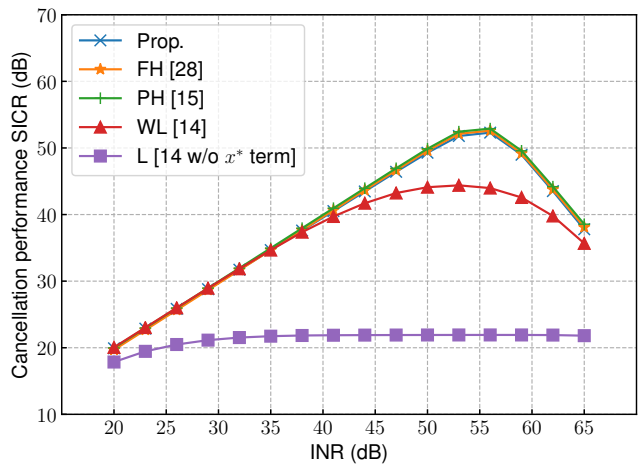

Fig. 6. The median SICR value of each canceller with the LS algorithm at $N_{\mathrm{Tr}}=60$. The smoothness factor of the LNA is $S_{\mathrm{LNA}}=1$. For the proposed scheme, $\beta=-20 \mathrm{~dB}$ and $\gamma=2 \mathrm{~dB}$.

confirmed from Fig. 2 and Fig. 3. Additionally, it is shown that cancellation performance is increased if the image margin $\beta$ is increased. The reason is that the estimated power of the $(p, q)$ th basis function is increased with image margin $\beta$, and then the set of basis functions for training $\mathcal{J}[k]$ becomes larger with increasing $\beta$ as shown in (56)-(58). In the following simulations, we use $\beta=-20 \mathrm{~dB}$ and $\gamma=2 \mathrm{~dB}$ because the proposed scheme with these parameters does not once achieve a SICR of less than $47 \mathrm{~dB}$ in all trials as we can see in Fig. 3.

In Fig. 5, the median cancellation performance of all trials on each canceller with the LS algorithm is shown at $N_{\operatorname{Tr}}=60$

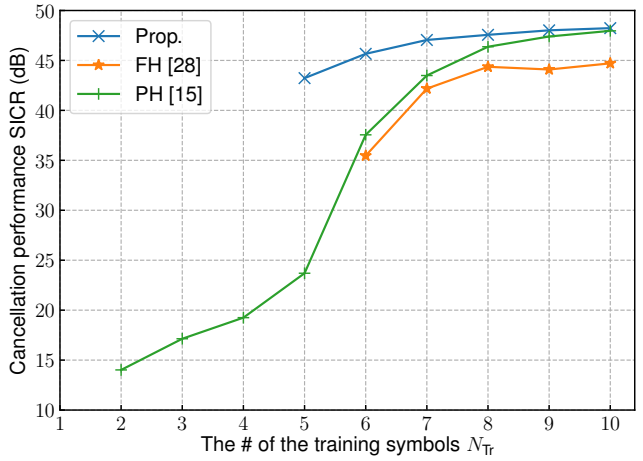

Fig. 7. The convergence of each canceller with the LS algorithm at INR $=50 \mathrm{~dB}$. SICR values are median values of all trials at each situation. The smoothness factor of the LNA is $S_{\mathrm{LNA}}=3$. For the proposed scheme, $\beta=$ $-20 \mathrm{~dB}$ and $\gamma=2 \mathrm{~dB}$.

and $S_{\mathrm{LNA}}=3$. The time-domain linear canceller, which is implemented based on [14] without the conjugated term $x^{*}$, cannot reconstruct the nonlinear self-interference signal. Hence the cancellation performance of the linear canceller is saturated at about $22 \mathrm{~dB}$. For a similar reason, the cancellation performance of the widely linear canceller [14], which can reconstruct both the linear term $x$ and the conjugated term $x^{*}$ only, is saturated at about $45 \mathrm{~dB}$. In contrast, the nonlinear cancellers such as the augmented nonlinear canceller [15], the frequency-domain Hammerstein canceller [28], and the proposed scheme achieved an SICR of about $50 \mathrm{~dB}$ at INR of $50 \mathrm{~dB}$. Hence, by the proposed basis function selection technique, the self-interference cancellation performance of the frequency-domain Hammerstein canceller hardly decreases.

In Fig. 6, the median cancellation performance of all trials on each canceller with the LS algorithm is shown at $N_{\mathrm{Tr}}=60$ and $S_{\mathrm{LNA}}=1$. In this situation, we simulate the LNA as a highly nonlinear component like the PA of the transmitter. Even when $S_{\mathrm{LNA}}=1$, we can confirm that the proposed method achieves the same cancellation performance as a conventional time-domain canceller. Thus, cancellation performance is not degraded by the proposed selection technique even if nonlinearity of the LNA appears strongly. When INR $>53 \mathrm{~dB}$, the cancellation performance of all cancellers simulated in this paper degrades because they cannot remove nonlinear signals generated by the LNA. The slope of the degradation is -2 [SICR $\mathrm{dB} /$ INR $\mathrm{dB}$ ] because we can write the SICR with a strong nonlinear signal $d_{\mathrm{LNA}}[n]$ generated by the LNA as

$$
\mathrm{SICR} \approx \frac{\mathbb{E}\left[|y[n]|^{2}\right]}{\mathbb{E}\left[\left|z[n]+d_{\mathrm{LNA}}[n]\right|^{2}\right]}=\mathcal{O}\left(\frac{I}{N+I^{3}}\right)=\mathcal{O}\left(I^{-2}\right),
$$

where $I$ is the power of self-interference signal. There are few papers which consider nonlinearities of the LNA such as [16], [19]. We will attack the nonlinearity of the LNA as a future work since it is challenging to describe a signal model that includes the nonlinearity of the LNA.

In Fig. 7, the convergence performance of each canceller with the LS algorithm is shown at INR $=50 \mathrm{~dB}$. In Fig. 7, 


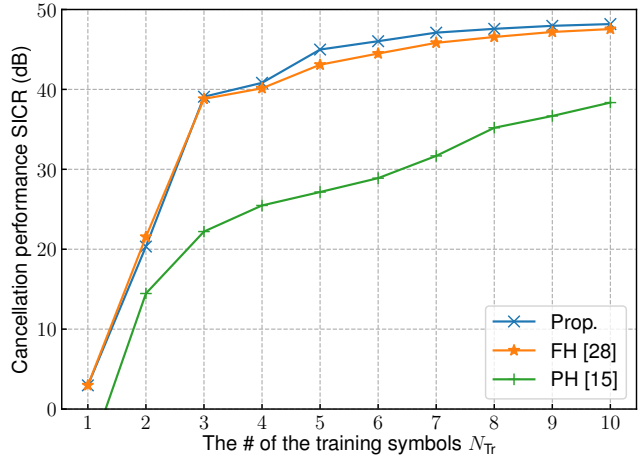

Fig. 8. The convergence of each canceller with RLS algorithm at INR = $50 \mathrm{~dB}$. SICR values are median values of all trials at each situation. The smoothness factor of the LNA is $S_{\mathrm{LNA}}=3$. For the proposed scheme, $\beta=$ $-20 \mathrm{~dB}$ and $\gamma=2 \mathrm{~dB}$.

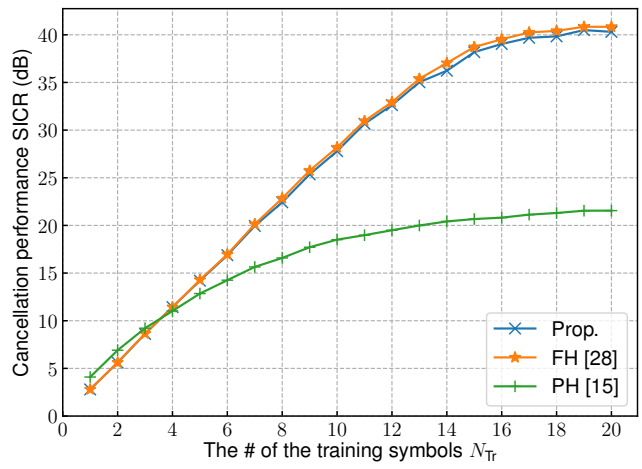

Fig. 9. The convergence of each canceller with NLMS algorithm at INR $=50 \mathrm{~dB}$. SICR values are median values of all trials at each situation. The smoothness factor of the LNA is $S_{\mathrm{LNA}}=3$. For the proposed scheme, $\beta=$ $-20 \mathrm{~dB}$ and $\gamma=2 \mathrm{~dB}$.

we can find that the proposed technique improves the initial convergence speed of the frequency-domain Hammerstein canceller, and it achieves better cancellation performance than the conventional method when the number of training symbols $N_{\operatorname{Tr}}$ is less than eight. By removing the basis functions unnecessary for self-interference cancellation by the proposed technique, the number of parameters of the canceller decreases, and convergence performance is improved. In Fig. 8, the convergence performance of each canceller with RLS algorithm is shown at INR $=50 \mathrm{~dB}$. In contrast with the case of the LS algorithm, the frequency-domain cancellers predominantly show better convergence performance than the time-domain nonlinear canceller. In the time domain, the input signal of the canceller is strongly colored, and convergence speed of an adaptive algorithm decreases with a colored input signal. However, the input signal of an adaptive algorithm of the frequency-domain Hammerstein canceller is almost white, and the convergence speed of them is faster than that seen in the time-domain case. In Fig. 9, the convergence performance of each canceller with the NLMS algorithm is shown at $\mathrm{INR}=50 \mathrm{~dB}$. As with the RLS algorithm, the frequencydomain cancellers show better convergence performance than the time-domain nonlinear canceller.

Fig. 10 shows the computational cost of the training stage at different INR, and Fig. 11 shows the selected percentage

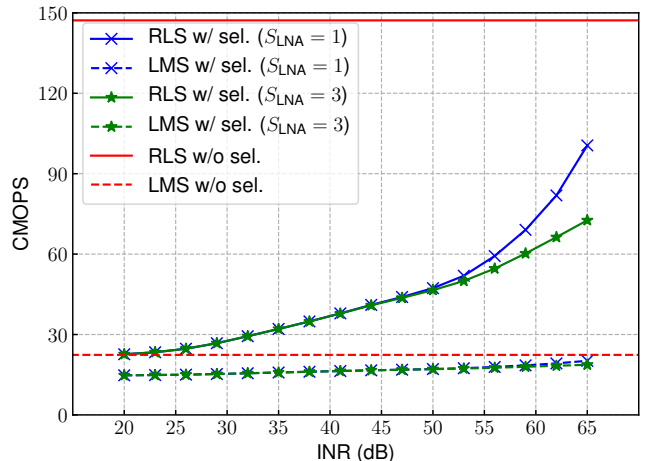

Fig. 10. The computational cost at a training stage with $\beta=-20 \mathrm{~dB}$ and $\gamma=2 \mathrm{~dB}$.

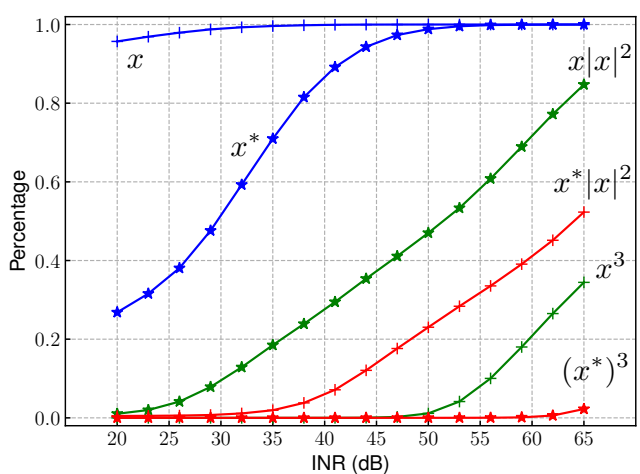

Fig. 11. The selected percentage in all subcarriers for each basis function In this figure, $\beta=-20 \mathrm{~dB}, \gamma=2 \mathrm{~dB}$, and $S_{\mathrm{LNA}}=3$.

TABLE II

CMOPS OF TIME-DOMAIN CANCELLERS

\begin{tabular}{c|c}
\hline \hline Canceller & CMOPS \\
\hline Linear with NLMS & 128 \\
\hline Linear with RLS & $1.66 \times 10^{4}$ \\
\hline Widely Linear with NLMS & 256 \\
\hline Widely Linear with RLS & $6.60 \times 10^{4}$ \\
\hline Parallel Hammerstein with NLMS & 768 \\
\hline Parallel Hammerstein with RLS & $5.90 \times 10^{5}$ \\
\hline \hline
\end{tabular}

in all subcarriers for each basis function on the proposed selection technique. When the proposed selection technique is disabled, the CMOPS of the RLS case is about 147 at all INR. On the other hand, using the basis function selection, the necessary basis functions are selected according to the INR, which can be found in Fig. 11, and, as a result, computational cost is reduced. Since the computational complexity of the RLS algorithm increases with the square of the number of basis functions, it is susceptible to the effect of the selection technique, but the effect for the NLMS is small because the computational complexity of the NLMS algorithm increases linearly. When INR is $20 \mathrm{~dB}$, the computational cost of the RLS case is less than $1 / 5$ as compared with a case without the selection, and even when INR is $50 \mathrm{~dB}$, it is less than half. When INR is greater than $50 \mathrm{~dB}$, the difference of the CMOPS between $S_{\mathrm{LNA}}=1$ and $S_{\mathrm{LNA}}=3$ is gradually increased. The proposed technique assumes that the LNA is a linear component, and basis functions cannot be selected correctly when the nonlinearity of the LNA is greater than the noise. In TABLE II, CMOPS values of time-domain cancellers are 


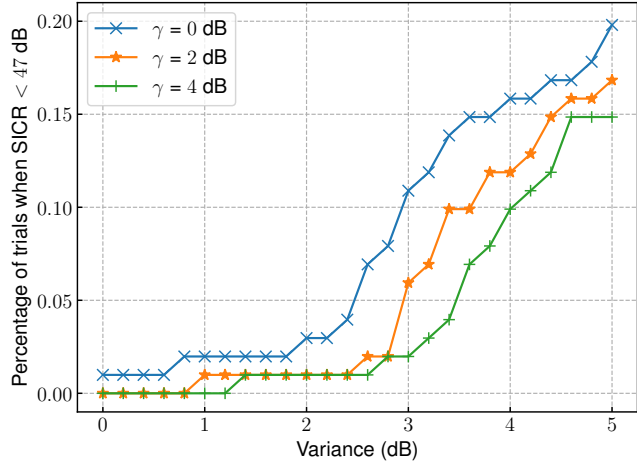

Fig. 12. The percentage of trails where the SICR of the proposed scheme is lower than $47 \mathrm{~dB}$ in all trials when nonlinear parameters are varied on log-normal distribution. In this simulation, the LS algorithm is used as the training algorithm. INR, $\beta, \gamma$, and $S_{\mathrm{LNA}}$ are $50 \mathrm{~dB},-20 \mathrm{~dB}, 2 \mathrm{~dB}$, and 3 respectively.

shown. Compared with the time-domain Hammerstein canceller optimized by the RLS algorithm, the proposed canceller achieves about $1.2 \times 10^{4}$ times lower computational cost on the training stage at INR $=50 \mathrm{~dB}$. When the NLMS algorithm is used, the proposed canceller achieves about 45 times lower computational cost than the time-domain Hammerstein canceller at INR $=50 \mathrm{~dB}$.

At the end of the simulation evaluation, we check how the self-interference cancellation performance of the proposed scheme is affected by fluctuations of the transmitter characteristics. Fig. 12 shows the percentage of trails in which the cancellation performance of the proposed scheme is lower than $47 \mathrm{~dB}$ in all trials at INR $=50 \mathrm{~dB}$ when log-normally distributed fluctuations are given to transmit power, IRR, IIP3 of the PA, and the gain of the PA independently. In other words, at the premeasurement stage we assign the values shown in TABLE I to each parameter, and at the training stage we assign log-normally distributed independent random variables to each parameter, respectively. It is found that the proposed scheme with $\gamma=2 \mathrm{~dB}$ is robust against fluctuations of $0.8 \mathrm{~dB}$ in variance. Generally, these parameters are dependent on temperature and supply voltage and vary by only about $1 \mathrm{~dB}$ at the maximum. Therefore, it can be seen that the proposed scheme is sufficiently effective against temporal characteristic fluctuations of equipment.

\section{CONCLUSION}

In this paper, we have proposed a basis function selection technique of the frequency-domain Hammerstein selfinterference canceller for in-band full-duplex communication systems. The estimation technique of the power spectral density of the received self interference is developed from the detailed nonlinear characteristics of a full-duplex terminal. The proposed selection technique reduces unnecessary basis functions for cancellation before the training stage according to the estimated self-interference power at each discrete frequency. Simulation results show that the proposed technique improves computational cost and convergence performance of the original frequency-domain Hammerstein canceller. It is shown that computational cost can be reduced to about one- fifth in the low self-interference situation by reducing the basis functions according to the estimated self-interference signal power. In addition, by the proposed basis function selection technique, self-interference cancellation performance of the frequency-domain Hammerstein canceller hardly decreases and achieves similar cancellation performance compared with the original.

\section{APPENDIX}

\section{A. Derivation of (53) and (54)}

We can assume that $\Psi_{p_{1}, q_{1}, i}^{\mathrm{SWP}}[k]$ and $\Psi_{p_{2}, q_{2}, i}^{\mathrm{SWP}}[k]$ are independent when $\left(p_{1}, q_{1}\right) \neq\left(p_{2}, q_{2}\right)$, because they are sums of a large number of combinations of subcarriers as (19). When $\Psi_{p_{1}, q_{1}, i}^{\mathrm{SWP}}[k]$ and $\Psi_{p_{2}, q_{2}, i}^{\mathrm{SWP}}[k]$ are independent, $\mathbb{E}\left[\left|Y_{\mathrm{VGA}}^{\mathrm{SWP}}[k]\right|^{2}\right]$ can be written as follows:

$$
\begin{aligned}
\mathbb{E}\left[\left|Y_{\mathrm{VGA}}^{\mathrm{SWP}}[k]\right|^{2}\right] & =\sum_{p, q}^{\infty} \mathbb{E}\left[\left|a_{p+q} c_{p, q} H[k] \Psi_{p, q}^{\mathrm{SWP}}[k]\right|^{2}\right] \\
& =\mathbb{E}\left[\left|a_{1} H[k] \Psi_{1,0}^{\mathrm{SWP}}[k]\right|^{2}\right] \\
& +\underbrace{\sum_{(p, q) \neq(1,0)}^{\infty} \mathbb{E}\left[\left|a_{p+q} c_{p, q} H[k] \Psi_{p, q}^{\mathrm{SWP}}[k]\right|^{2}\right]}_{\geq 0},
\end{aligned}
$$

Thus, the following inequation is derived as:

$$
\mathbb{E}\left[\left|a_{1} H[k] \Psi_{1,0}^{\mathrm{SWP}}[k]\right|^{2}\right] \leq \mathbb{E}\left[\left|Y_{\mathrm{VGA}}^{\mathrm{SWP}}[k]\right|^{2}\right] .
$$

Transforming (4) with respect to $Y_{\mathrm{VGA}}^{\mathrm{SWP}}[k]$, (87) is derived.

$$
Y_{\mathrm{VGA}}^{\mathrm{SWP}}[k]=\frac{Y_{i}^{\mathrm{SWP}}[k]-b^{\mathrm{RX}}\left(Y_{i}^{\mathrm{SWP}}[-k]\right)^{*}}{1-\left|b^{\mathrm{RX}}\right|^{2}} .
$$

Finally, we derive (53) and (54) from (86) by the triangle inequality.

\section{REFERENCES}

[1] J. I. Choi, M. Jain, K. Srinivasan, P. Levis, and S. Katti, "Achieving single channel, full duplex wireless communication," in Proc. 16th Annu. Int. Conf. Mobile Comput. Netw., Sept. 2010, pp. 1-12.

[2] D. Kim, H. Lee, and D. Hong, "A survey of in-band full-duplex transmission: From the perspective of PHY and MAC layers," IEEE Commun. Surveys Tuts., vol. 17, no. 4, pp. 2017-2046, Fourthquarter 2015.

[3] D. Bharadia, E. McMilin, and S. Katti, "Full duplex radios," in Proc. ACM SIGCOMM, Aug. 2013.

[4] M. Jain, J. I. Choi, T. Kim, D. Bharadia, S. Seth, K. Srinivasan, P. Levis, S. Katti, and P. Sinha, "Practical, real-time, full duplex wireless," in Proc. 17th Annu. Int. Conf. Mobile Comput. Netw., Sept. 2011, pp. 301312.

[5] E. Everett, A. Sahai, and A. Sabharwal, "Passive self-interference suppression for full-duplex infrastructure nodes," IEEE Trans. Wireless Commun., vol. 13, no. 2, pp. 680-694, Feb. 2014.

[6] J. Tamminen, M. Turunen, D. Korpi, T. Huusari, Y. S. Choi, S. Talwar, and M. Valkama, "Digitally-controlled RF self-interference canceller for full-duplex radios," in Proc. 24th EUSIPCO, Aug. 2016, pp. 783-787.

[7] M. Heino, D. Korpi, T. Huusari, E. Antonio-Rodriguez, S. Venkatasubramanian, T. Riihonen, L. Anttila, C. Icheln, K. Haneda, R. Wichman, and M. Valkama, "Recent advances in antenna design and interference cancellation algorithms for in-band full duplex relays," IEEE Commun. Mag., vol. 53, no. 5, pp. 91-101, May 2015.

[8] M. Duarte, C. Dick, and A. Sabharwal, "Experiment-driven characterization of full-duplex wireless systems," IEEE Trans. Wireless Commun., vol. 11, no. 12, pp. 4296-4307, Dec. 2012. 
[9] J. Zhou, T. H. Chuang, T. Dinc, and H. Krishnaswamy, "Integrated wideband self-interference cancellation in the RF domain for FDD and full-duplex wireless," IEEE J. Solid-State Circuits, vol. 50, no. 12, pp. 3015-3031, Dec. 2015.

[10] J.-H. Lee, J. won Choi, J.-H. Jung, S.-C. Kim, and Y.-H. Kim, "Analog cancellation for full-duplex wireless in multipath self-interference channels," IEICE Trans. Commun., vol. E98.B, no. 4, pp. 646-652, 2015.

[11] M. Sakai, H. Lin, and K. Yamashita, "Adaptive cancellation of selfinterference in full-duplex wireless with transmitter IQ imbalance," in Proc. IEEE GLOBECOM, Dec. 2014, pp. 3220-3224.

[12] D. Korpi, Y. S. Choi, T. Huusari, L. Anttila, S. Talwar, and M. Valkama, "Adaptive nonlinear digital self-interference cancellation for mobile inband full-duplex radio: Algorithms and RF measurements," in Proc. IEEE GLOBECOM, Dec. 2015.

[13] L. Anttila, D. Korpi, V. Syrjälä, and M. Valkama, "Cancellation of power amplifier induced nonlinear self-interference in full-duplex transceivers," in Proc. Asilomar Conf. on Signals, Syst. Comput., Nov. 2013, pp. 1193 1198.

[14] D. Korpi, L. Anttila, V. Syrjälä, and M. Valkama, "Widely linear digital self-interference cancellation in direct-conversion full-duplex transceiver," IEEE J. Sel. Areas Commun., vol. 32, no. 9, pp. 16741687, Sept. 2014.

[15] D. Korpi, T. Huusari, Y. S. Choi, L. Anttila, S. Talwar, and M. Valkama, "Digital self-interference cancellation under nonideal RF components: Advanced algorithms and measured performance," in Proc. IEEE SPAWC, June 2015, pp. 286-290.

[16] D. Korpi, S. Venkatasubramanian, T. Riihonen, L. Anttila, S. Otewa, C. Icheln, K. Haneda, S. Tretyakov, M. Valkama, and R. Wichman, "Advanced self-interference cancellation and multiantenna techniques for full-duplex radios," in Proc. Asilomar Conf. on Signals, Syst. Comput., Nov. 2013, pp. 3-8.

[17] A. E. Nordsjo and L. H. Zetterberg, "Identification of certain timevarying nonlinear Wiener and Hammerstein systems," IEEE Trans. Signal Process., vol. 49, no. 3, pp. 577-592, Mar. 2001

[18] A. Guerin, G. Faucon, and R. L. Bouquin-Jeannes, "Nonlinear acoustic echo cancellation based on Volterra filters," IEEE Trans. Speech Audio Process., vol. 11, no. 6, pp. 672-683, Nov. 2003.

[19] E. Ahmed, A. M. Eltawil, and A. Sabharwal, "Self-interference cancellation with nonlinear distortion suppression for full-duplex systems," in Proc. Asilomar Conf. on Signals, Syst. Comput., Nov. 2013, pp. 11991203.

[20] — , "Self-interference cancellation with phase noise induced ICI suppression for full-duplex systems," in Proc. IEEE GLOBECOM, Dec. 2013, pp. 3384-3388.

[21] H. Lee, J. Choi, D. Kim, and D. Hong, "Impact of time and frequency misalignments in OFDM based in-band full-duplex systems," in Proc. IEEE WCNC, Mar. 2017.

[22] M. S. Amjad and O. Gurbuz, "Linear digital cancellation with reduced computational complexity for full-duplex radios," in Proc. IEEE WCNC, Mar. 2017.

[23] E. Ahmed and A. M. Eltawil, "All-digital self-interference cancellation technique for full-duplex systems," IEEE Trans. Wireless Commun., vol. 14, no. 7, pp. 3519-3532, July 2015.

[24] D. Korpi, L. Anttila, and M. Valkama, "Reference receiver based digital self-interference cancellation in MIMO full-duplex transceivers," in Proc. GC Wkshps, Dec. 2014, pp. 1001-1007.

[25] S. Li and R. D. Murch, "An investigation into baseband techniques for single-channel full-duplex wireless communication systems," IEEE Trans. Wireless Commun., vol. 13, no. 9, pp. 4794-4806, Sept. 2014.

[26] T. Riihonen, S. Werner, and R. Wichman, "Mitigation of loopback selfinterference in full-duplex MIMO relays," IEEE Trans. Signal Process., vol. 59, no. 12, pp. 5983-5993, Dec. 2011.

[27] H. Q. Ngo, H. A. Suraweera, M. Matthaiou, and E. G. Larsson, "Multipair full-duplex relaying with massive arrays and linear processing," IEEE J. Sel. Areas Commun., vol. 32, no. 9, pp. 1721-1737, Sept. 2014.

[28] K. Komatsu, Y. Miyaji, and H. Uehara, "Frequency-domain Hammerstein self-interference canceller for in-band full-duplex OFDM systems," in Proc. IEEE WCNC, Mar. 2017.

[29] M. Valkama and M. Renfors, "Digital filter design for I/Q imbalance compensation," in Proc. 10th EUSIPCO, Sept. 2000.

[30] W. H. Press, S. A. Teukolsky, W. T. Vetterling, and B. P. Flannery, Numerical Recipes 3rd Edition: The Art of Scientific Computing, 3rd ed. New York, USA: Cambridge University Press, 2007.

[31] Maxim Integrated, "MAX2242: $2.4 \mathrm{GHz}$ to $2.5 \mathrm{GHz}$ linear power amplifier," San Jose, CA, USA.

[32] — , "MAX2692/MAX2695: WLAN/WiMAX low-noise amplifiers," San Jose, CA, USA.
[33] D. Korpi, T. Riihonen, V. Syrjälä, L. Anttila, M. Valkama, and R. Wichman, "Full-duplex transceiver system calculations: Analysis of ADC and linearity challenges," IEEE Trans. Wireless Commun., vol. 13, no. 7, pp. 3821-3836, July 2014.

[34] C. Rapp, "Effects of HPA-nonlinearity on a 4-DPSK/OFDM-signal for a digital sound broadcasting system," Proc. Eur. Conf. Satellite Commun., pp. 179-184, Oct. 1991.

[35] J. Joung, C. K. Ho, K. Adachi, and S. Sun, "A survey on poweramplifier-centric techniques for spectrum- and energy-efficient wireless communications," IEEE Commun. Surveys Tuts., vol. 17, no. 1, pp. 315333, Firstquarter 2015.

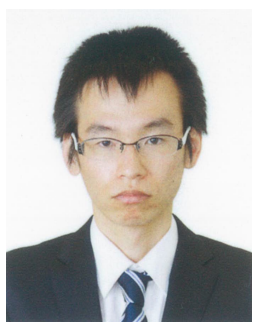

Kazuki Komatsu (S'17) was born in Nara Prefecture, Japan, on January 10, 1994. He received the B.E. and M.E. degrees from Toyohashi University of Technology, Japan, in 2016 and 2018, respectively. $\mathrm{He}$ is currently a master course student in the Graduate School of Engineering, Toyohashi University of Technology, Japan. He received the IEEE Nagoya Section Excellent Student Award in 2016 and the IEEE Communications Society Young Professionals Best Paper Award in 2017. His current research interests include in-band full-duplex communications.

$\mathrm{He}$ is a member of IEICE.

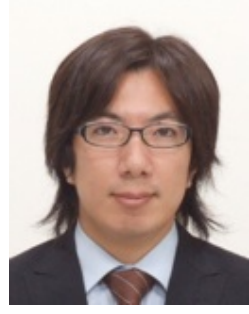

Yuichi Miyaji (S'10-M'13) was born in Kagoshima Prefecture, Japan, on July 13, 1984. He received the B.E., M.E., and Ph.D. degrees from Toyohashi University of Technology, Japan, in 2007, 2009, and 2013, respectively. Since 2013, he has been an Assistant Professor in the Department of Electrical and Electronic Information Engineering, Toyohashi University of Technology, Japan. Dr. Miyaji received the IEICE Young Researchers Award and the IEICE Communications Society: Distinguished Contributions Award in 2016. His current research interests include wireless networks and in-band full-duplex communications. He is a member of IEICE and IPSJ.

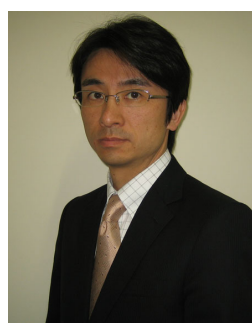

Hideyuki Uehara (S'92-M'97) received his B.E., M.E., and Ph.D. degrees in electrical engineering from Keio University, Yokohama, Japan, in 1992, 1994, and 1997, respectively. Since 1997, he has been with Toyohashi University of Technology, Japan, where he is currently a Professor of the Department of Electrical and Electronic Information Engineering. From 2002 to 2003, he was also a Visiting Researcher at the Advanced Telecommunications Research Institute International (ATR), Kyoto, Japan. His current research interests include wireless access and multihop communications. He served as an Editor of the Editorial Committee of IEICE Transactions on Communications from 2010 to 2011, and also as a Vice Chair of the Editorial Board of IEICE Communications Society from 2014 to 2015 . From 2017 he has been serving as a Treasurer of the IEEE Nagoya Section. He received the Distinguished Contributions Award of IEICE Communications Society in 2002, 2006, 2011, 2012, and 2016. Dr. Uehara is a Member of IEEE and IPSJ. 